
La red de operadores de Sivigila está compuesta por todas aquellas Unidades Primarias Generadoras de Datos UPGD y Unidades Informadoras UI que se encuentran caracterizadas en Sivigila.

En periodo 13 del año 2020, hay un total de 5168 UPGD y 7258 UI activas en Sivigila. Al observar la línea base del año 2012 y durante los años posteriores, las UPGD tuvieron un comportamiento ascendente hasta el año 2015, posteriormente, se observa un descenso sostenido hasta el año 2019 y de allí un leve aumento frente al periodo XIII del año 2020 (22 operadores). Se debe tener en cuenta que la tendencia de las UPGD es a la disminución a final de año debido al cierre temporal de algunas por las festividades y vacaciones de fin de año. Con respecto a las unidades informadoras (UI), la tendencia ha sido al aumento año tras año, teniendo como línea de base en el año 2012, 1244 UI y un incremento del $83 \%$ en el 2020 llegando a 7258 UI activas. Este incremento es debido al trabajo anual que realizan las entidades territoriales de verificar y visitar los potenciales operadores que han sido extraídos del Registro Especial de Prestadores de Servicios de Salud (REPS) y que ha permitido la ampliación de la red (figura 1).

Figura 1. Comportamiento de la red de operadores Colombia 2012- periodo XIII 2020

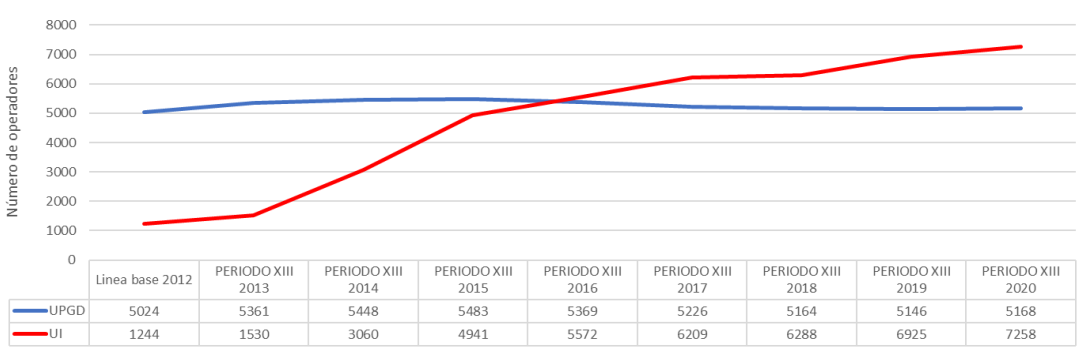

Fuente: portal Sivigila 2020

Al comparar la red de UPGD de los años 2019 y 2020, se evidencia una disminución significativa a partir del periodo 3 del 2020, que coincide con el inicio del aislamiento obligatorio y por ende el cierre e inactivación en Sivigila de UPGD caracterizadas por la emergencia sanitaria de la pandemia Covid-19. Hay que resaltar que la mayoría de las instituciones que se inactivaron son del primer nivel de atención. A partir del periodo 7 de 2020, se observa un incremento en el número de UPGD activas con respecto a periodos anteriores (5168 UPGD activas en periodo 13), que coincide con la reapertura parcial de algunos sectores económicos en el país, cerrando incluso con un número mayor de entidades activas que la observadas en el periodo XIII de 2010.

Figura 2. Comportamiento de los operadores caracterizados como UPGD año 2019 y periodo XIII de 2020

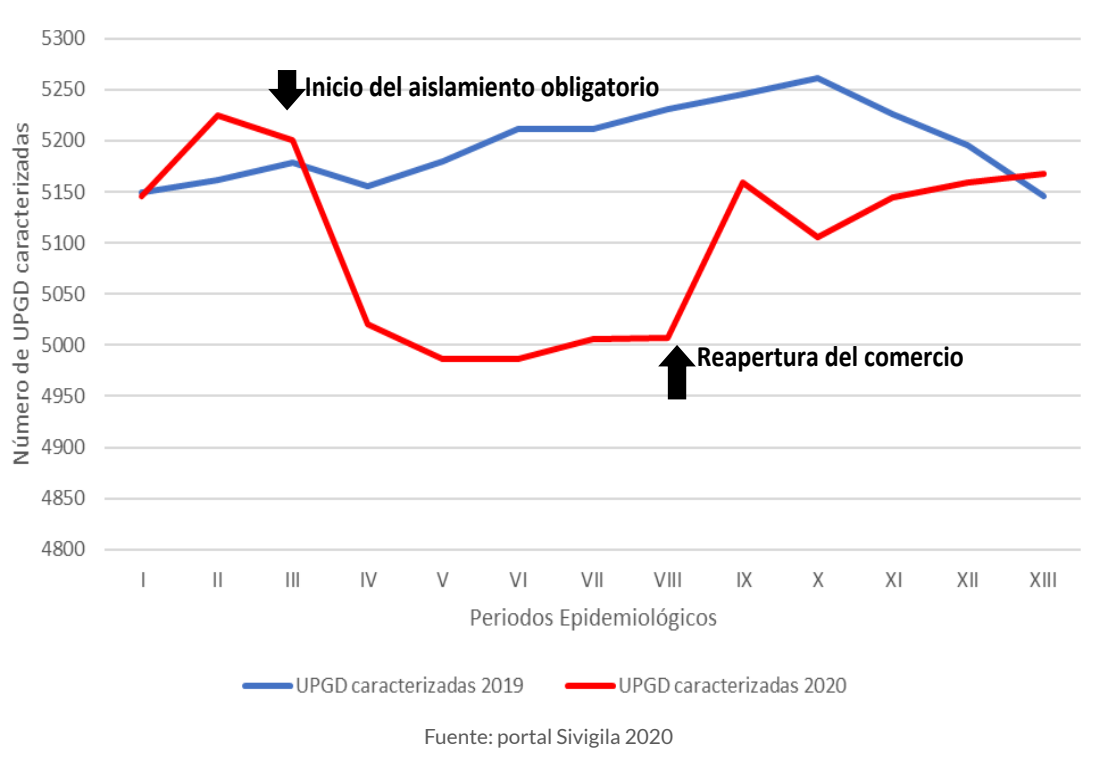

Según lineamientos establecidos por el INS, en el cuarto trimestre del 2020 , las entidades territoriales deben tener un cumplimiento de visitas a potenciales UPGD del $100 \%$, e ingresar los soportes en el portal gidos deberán ser caracterizadas como UPGD y realizar una primera notificación negativa para quedar activas en el sistema. En el año 2020, se seleccionaron del REPS, 1.072 potenciales operadores que se clasificaron presuntivamente como Unidades Primarias Generadoras de Daweb de Sivigila 4.0, las instituciones que cumplan con los requisitos exi-

Tema central
Situación Nal.
Mortalidad
Trazadores
Brotes
COVID-19
Tablas

La información es notificada semanalmente por las entidades territoriales (ET) al Instituto Nacional de Salud (INS) a través del Sistema de vigilancia en salud pública (Sivigila). El número de casos, puede variar después de que se realizan unidades de análisis en las ET para el ajuste y la clasificación de caso en cada evento. 
tos (UPGD), al mes de diciembre, 30\% de las entidades territoriales han cumplido con la meta de visitar el 100\% de las potenciales UPGD, entre la que se encuentran Amazonas, Arauca, Caquetá, Cartagena, Casanare, Cundinamarca, Guainía, Huila, Meta, Norte De Santander, Putumayo.

De los 37 departamentos y distritos en el país, 11 (30\%) entidades disminuyeron el número de UPGD activas en el sistema.

Con respecto al comportamiento de las UPGD caracterizadas con respecto al año 2019, se observa que San Andrés, Santa Marta, Córdoba y Vichada son las entidades que presentaron mayor porcentaje de aumento, mientras que Vaupés, Magdalena, Guainía, Arauca y Putumayo presentaron el mayor porcentaje de disminución. En este sentido con la llegada del virus pandémico se observó que algunas UPGD cambiaron su caracterización a UI debido a la disminución en el número de consultas o se realizó el cierre de algunas de ellas como se observó en la gráfica anterior.

Tabla 1. Comportamiento de los operadores caracterizados como UPGD por entidad territorial a periodo XIII, 2019 y 2020
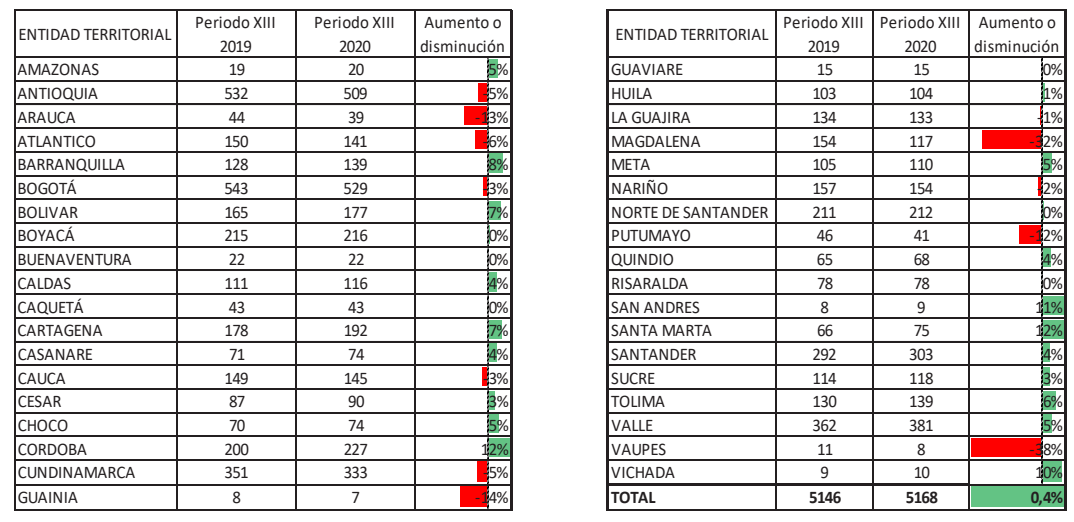

Fuente: Portal Sivigila 2020
Con respecto a las UI, se observa un comportamiento contrario al de las UPGD, en tanto al hacer un comparativo con el año 2019, se observa incremento en el número de UI activas en el sistema, pasando de 6925 UI activas en el 2019, a 7258 en periodo 13 de 2020, con un aumento del $5 \%$. Se debe tener en cuenta que a raíz de la pandemia se ha realizado la caracterización de nuevos operadores como UI para la atención y toma de muestra en la emergencia Covid-19, entre los que se encuentran los laboratorios de procesamiento de muestras que a la fecha se encuentran caracterizados en SIVIGILA, aportando de esta forma a la vigilancia y notificación de este evento.

Figura 3. Comportamiento de operadores caracterizados como UI año 2019 y a periodo XIII de 2020

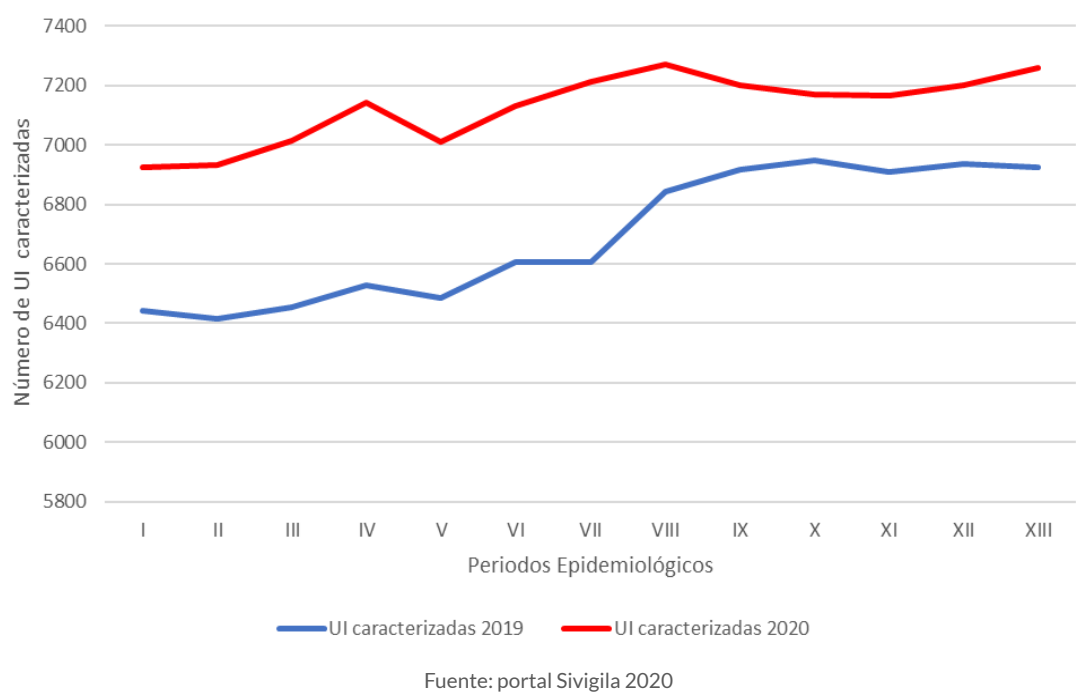

Tema central

Situación Nal.

Mortalidad

Trazadores

Brotes

COVID-19

Tablas 
En cuanto a las potenciales UI, para el 2020 se seleccionaron del REPS un total de 10.130 instituciones que fueron tentativamente clasificados como Unidades Informadoras (UI) de acuerdo con parámetros establecidos. Al mes de diciembre, $14 \%$ de las entidades territoriales han cumplido con la meta de visitar el $100 \%$ de las potenciales UI en cuarto trimestre, entre la que se encuentran Arauca, Córdoba, Meta, Putumayo y Vichada.

De los 37 departamentos y distritos en el país, 28 entidades (76\%) aumentaron el número de UI activas en el sistema, al comparar las UI activas en periodo 12 de 2019 y 2020 muestra mayor porcentaje de aumento en los departamentos Huila, Magdalena y Santa Marta en tanto algunas de ellas eran UPGD pero cambiaron su caracterización y otras son nuevas, que no se encontraban en REPS y empezaron a prestar sus servicios este año a raíz de la pandemia de COVID, como por ejemplo algunas cuyo objeto es la toma de muestras. En el caso del departamento de Huila por ejemplo, las ciudades que aumentaron su número de UI para toma de muestras fueron Neiva, Pitalito, Garzón y La Plata.

Departamentos como Antioquia, Cartagena y Amazonas presentaron los mayores porcentajes de disminución de estas entidades, las cuales en pandemia fueron inactivadas, especialmente los consultorios particulares, comisarías de familia entre otras que no podían operar durante esta etapa.
Tabla 2. Comportamiento de los operadores caracterizados como UI por entidad territorial a periodo XIII, 2019 y 2020
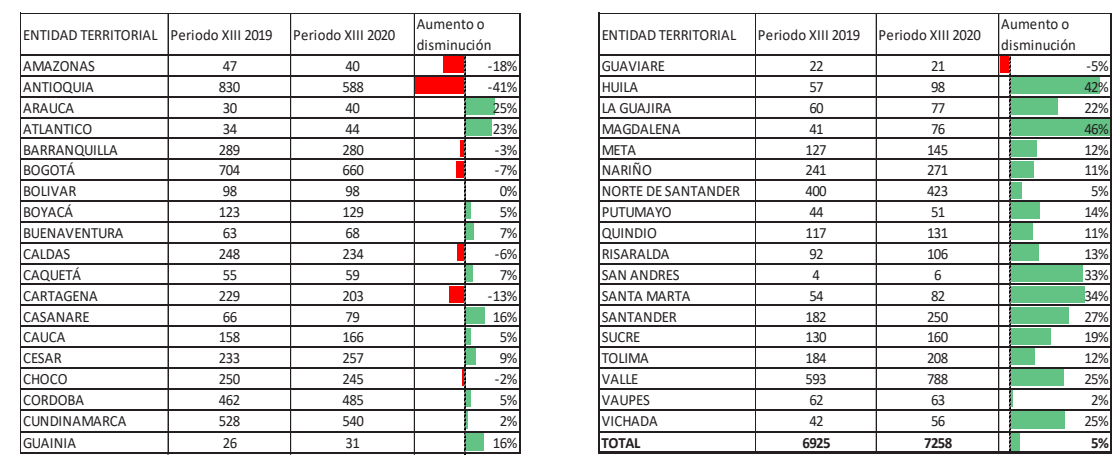

Fuente: portal Sivigila 2020

\section{Conclusiones y recomendaciones}

- En el último año la cantidad de UPGD ha disminuido debido al aislamiento obligatorio y las unidades informadoras han aumentado por la caracterización de laboratorios e IPS de atención de COVID.

- El comportamiento de las UPGD se observa más fluctuante que el de las UI en tanto son más susceptibles de cambiar su caracterización debido a factores externos que cambien el volumen de atención como las festividades y en el año 2020 debido a la pandemia.

- El proceso de ampliación de la red de operadores ha permitido monitorear la caracterización en SIVIGILA de las entidades habilitadas que son susceptibles de captar eventos de interés en salud pública año tras año, siendo una de las actividades más importantes que contribuyen a mantener la red actualizada, incluyendo en los últimos años laboratorios de patología y entidades que atienden enfermedades huérfanas.

- El portal web Sivigila 4.0 ha permitido a las entidades territoriales desarrollar el proceso de ampliación de la red de operadores de manera organizada y efectiva, al contar con la opción de adjuntar los soportes de las visitas que van realizando a lo largo del año. 


\section{SITUACIÓN NACIONAL}

\section{Tablero de control del análisis de datos de la vigilancia}

Para el análisis de los eventos de mayor notificación en el país, se compara el valor observado en la semana epidemiológica correspondiente, con una línea de base de referencia, que está conformada con la información de esos eventos reportados en 15 intervalos de tiempo de al menos cinco años anteriores.

Se identifica que en la semana epidemiológica 13 , los eventos de malaria, accidente ofídico, parotiditis, mortalidad perinatal y neonatal tardía, leishmaniasis cutánea, bajo peso al nacer y varicela se encuentran por debajo de lo esperado, los demás eventos están dentro del comportamiento histórico de la notificación (Figura 4).

Figura 4. Comparación de la notificación de casos de eventos priorizados, de alta frecuencia, según su comportamiento histórico. Colombia, semana epidemiológica 13 de 2021
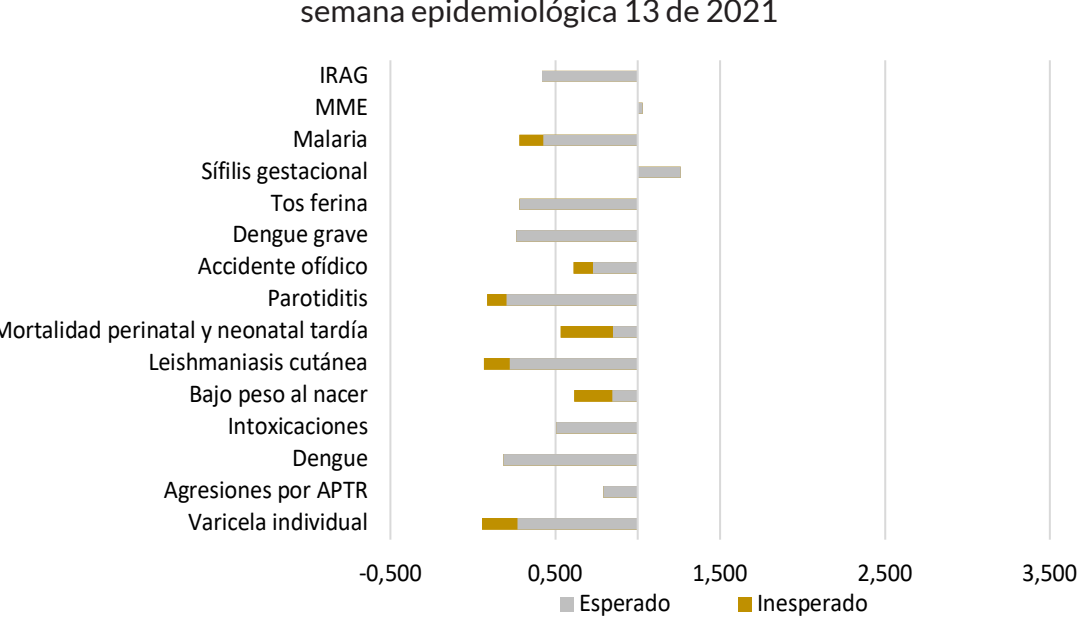

Fuente: Sivigila, Instituto Nacional de Salud, Colombia, 2021
Para el análisis de los eventos de baja frecuencia o raros, se comparan los casos observados y los esperados según su comportamiento histórico. Se calcula la probabilidad de la significación estadística de la comparación. Valores menores a 0,06 en la columna "Poisson" indican que existe una diferencia significativa entre lo observado y lo esperado para la semana analizada.

El evento de mortalidad por EDA 0-4 años se encuentra por encima del valor esperado, mientras que los eventos de leptospirosis, sarampión, síflis congénita, mortalidad por dengue y rubeola presentan una disminución significativa relacionada con el comportamiento de notificación histórico. Los demás eventos se encuentran dentro del comportamiento histórico (Tabla 3).

Tabla 3. Comparación de casos notificados de eventos priorizados, de baja frecuencia, según su comportamiento histórico, Colombia, semana epidemiológica 13 de 2021

\begin{tabular}{lrrr} 
Evento & Obser-vado & Esperado & Poisson \\
Mortalidad por EDA 0-4 Años & 3 & 1 & 0,00 \\
Leptospirosis & 10 & 30 & 0,00 \\
Sarampión & 13 & 63 & 0,00 \\
Síflis congénita & 19 & 24 & 0,00 \\
Mortalidad por dengue & 1 & 3 & 0,01 \\
Rubeola & 0 & 18 & 0,01 \\
EAV & 38 & 15 & 0,06 \\
Mortalidad materna & 8 & 7 & 0,08 \\
Mortalidad por IRA & 4 & 11 & 0,09 \\
Leishmaniasis mucosa & 0 & 1 & 0,09 \\
Lepra & 0 & 7 & 0,12 \\
Tuberculosis farmacorresistente & 4 & 9 & 0,13 \\
Fiebre tifoidea y paratifoidea & 0 & 3 & 0,24 \\
\hline
\end{tabular}

Fuente: Sivigila, Instituto Nacional de Salud, Colombia, 2021

\section{Cumplimiento en la notificación}

Para esta semana la notificación recibida por el Instituto Nacional de Salud correspondiente a las unidades notificadoras departamentales y distritales (UND) fue del $100 \%$, permaneció igual comparándola con la semana anterior, así como con la misma semana del 2020. 
El reporte de las unidades notificadoras municipales (UNM) a nivel nacional fue $100 \%$ (1117/ 1117 UNM), permaneció igual comparándolo con la semana anterior y también con respecto a la misma semana de 2020. El país cumplió con la meta del 97 \% en la notificación de UNM.

El cumplimiento de las Unidades Primarias Generadoras de Datos (UPGD) esta semana fue de 96,8 \% (5 006 / 5171 UPGD); disminuyo $0,1 \%$ comparándolo con la semana anterior y aumentó $2,2 \%$ con respecto a la misma semana de 2020.

El país cumplió con la meta para la notificación de UPGD, en la gráfica se refleja con la línea verde que es el porcentaje de notificación de la presente semana; la línea amarilla representa el número mínimo de UPGD que debe notificar (meta $90 \%$ ) y la línea azul evidencia la red actual, un total de 5171 UPGD caracterizadas (Figura 5).

Figura 5. Cumplimiento de la notificación por UPGD, Colombia, semanas epidemiológicas 08 a 13 de 2021

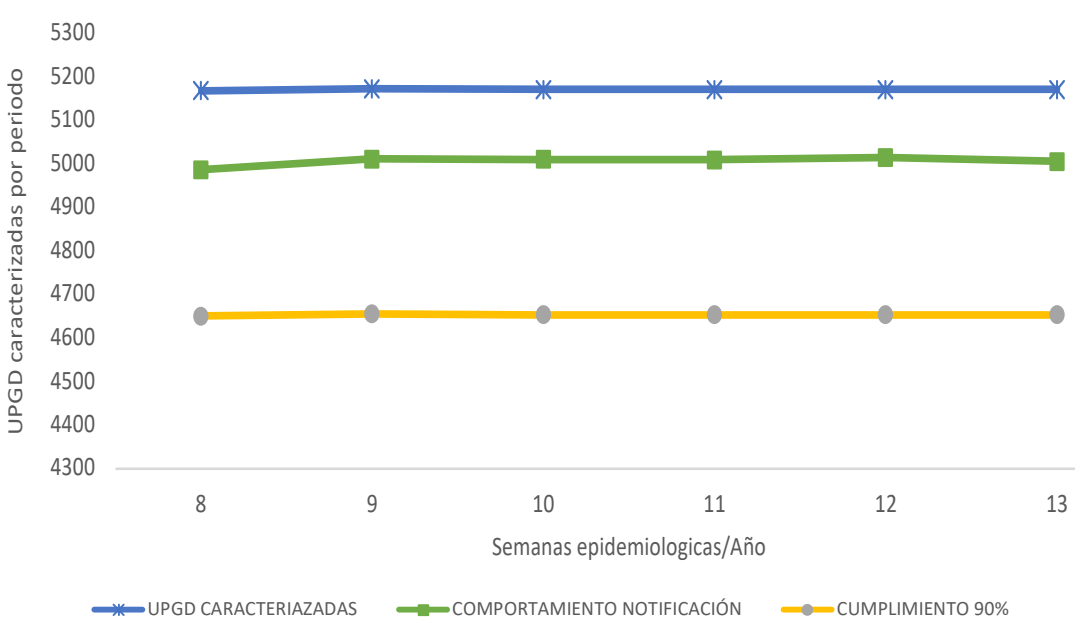

Fuente: Sivigila, Instituto Nacional de Salud, Colombia, 2021

\section{MORTALIDAD}

\section{Mortalidad en menores de 5 años por eventos priorizados}

Esta semana se notificaron 7 muertes en menores de 5 años: 4 probablemente asociadas a infección respiratoria aguda y 3 a enfermedad diarreica aguda, no se notificaron casos de muertes por desnutrición.

\section{Mortalidad por infección respiratoria aguda}

Se notificaron 4 muertes por infección respiratoria aguda en menores de 5 años, 2 correspondientes a esta semana y 2 de semanas anteriores. Para la misma semana epidemiológica de 2020 se notificaron 6 casos.

Para esta semana se observó un aumento en el número de casos en las entidades territoriales de Arauca y Guainía en comparación con el histórico notificado a semana epidemiológica 13 entre 2014 a 2020, mientras que, en Antioquia se observó una disminución. En las entidades territoriales restantes no se presentaron variaciones.

\section{Mortalidad por desnutrición}

Para esta semana no se notificaron casos de muertes probablemente asociadas a desnutrición. Para la misma semana epidemiológica de 2020 se notificaron 3 casos.

Para esta semana se observó un aumento en el número de casos en las entidades territoriales de La Guajira, Guainía, Santa Marta y Sucre en comparación con el histórico notificado a semana epidemiológica 13 entre 2014 a 2020. En las demás entidades no se observaron variaciones.

\section{Mortalidad por enfermedad diarreica aguda}

Para esta semana se notificaron 3 muertes probablemente asociadas a enfermedad diarreica aguda en menor de 5 años, 1 correspondiente a esta semana y 2 de semanas anteriores. Para la misma semana epidemiológica de 2020 se notificaron 2 casos.

Para esta semana se observó un aumento en el número de casos en las entidades territoriales de Amazonas, Atlántico, Casanare, Santander, Sucre
Tema centra

Situación $\mathrm{Nal}$

Mortalidad

Trazadores

Brotes

COVID-19

Tablas 
y Valle del Cauca, en comparación con el histórico notificado a semana epidemiológica 13 entre 2014 a 2020, mientras que, en Antioquia se observó una disminución. En las entidades territoriales restantes no se observaron variaciones.

Para el análisis de los datos se tomó el comportamiento de cada uno de los eventos a semana epidemiológica 13 entre 2014 a 2020 (histórico) y se comparó con los casos observados a la misma semana epidemiológica del 2021. La razón esperada siempre será 1 y la significancia estadística estará dada por el valor de $\mathrm{p}<0,05$ para identificar las entidades territoriales que presentan variaciones estadísticamente significativas.

\section{Mortalidad materna}

En la semana epidemiológica 13 de 2021 se notificaron 112 muertes maternas, 92 corresponden a mortalidad materna temprana (ocurridas durante el embarazo, parto y hasta los 42 días de terminada la gestación), 14 tardías (ocurridas desde el día 43 hasta un año de terminada la gestación) y 6 por causas coincidentes (lesiones de causa externa). Se observa un aumento del $21,0 \%$ en la mortalidad materna temprana respecto al 2020 (Tabla 4).

Tabla 4. Mortalidad materna según tipo de muerte, Colombia, semana epidemiológica 13, 2019 a 2021

\begin{tabular}{ccccc} 
& \multicolumn{3}{c}{ Tipo de muerte } & Total \\
\cline { 2 - 4 } 2019 & Temprana & Tardía & Coincidente & 129 \\
2020 & 77 & 34 & 18 & 128 \\
2021 & 76 & 44 & 8 & 112 \\
\hline \multicolumn{4}{c}{ Fuente: Sivigila, Instituto Nacional de Salud, Colombia, 2019-2021 }
\end{tabular}

La razón nacional preliminar de mortalidad materna a semana epidemiológica 13 es de 43,6 muertes por cada 100000 nacidos vivos. La razón de mortalidad materna superior a 100 muertes por cada 100 000 nacidos vivos se observó en las entidades territoriales de Guaviare, Chocó, Santa Marta, y Meta (Tabla 5).
Tabla 5. Razón de mortalidad materna según entidad territorial de residencia, Colombia, semana epidemiológica 13 de 2021

\begin{tabular}{lcc}
$\begin{array}{c}\text { Entidad territorial de } \\
\text { residencia }\end{array}$ & Número de casos & $\begin{array}{c}\text { Razón de MM por } 100000 \\
\text { nacidos vivos }\end{array}$ \\
Guaviare & 1 & 232,0 \\
Chocó & 3 & 177,4 \\
Santa Marta & 4 & 141,0 \\
Meta & 5 & 101,7 \\
Risaralda & 3 & 99,8 \\
Magdalena & 7 & 98,9 \\
Buenaventura & 1 & 74,2 \\
Putumayo & 1 & 68,9 \\
Córdoba & 5 & 65,7 \\
Cali & 5 & 64,6 \\
La Guajira & 4 & 60,3 \\
Antioquia & 11 & 48,3 \\
Sucre & 2 & 47,6 \\
Barranquilla & 3 & 46,4 \\
Cundinamarca & 5 & 44,8 \\
Colombia & 92 & 43,6 \\
Norte de Santander & 3 & 40,6 \\
Cartagena & 2 & 40,0 \\
Tolima & 2 & 39,8 \\
Caldas & 1 & 38,9 \\
Huila & 2 & 36,4 \\
Bogotá & 9 & 34,0 \\
Bolívar & 3 & 30,6 \\
Cesar & 2 & 28,6 \\
Valle del Cauca & 4 & 26,5 \\
Santander & 2 & 23,5 \\
Nariño & 1 & 19,5 \\
Atlántico & 1 & 17,3 \\
\hline
\end{tabular}

Tema central

Situación Nal.

Mortalidad

Trazadores

Brotes

COVID-19

Fuente: Sivigila, Instituto Nacional de Salud, Colombia, 2021 - DANE, Estadísticas Vitales preliminar 2020

Para la semana epidemiológica 13 de 2021 se observó un aumento significativo en las muertes maternas tempranas comparado con el promedio histórico en las entidades territoriales de Guaviare, Huila, Meta, Santa Marta y Cali y disminución en las entidades territoriales de Cauca y Nariño (Tabla 6).

Tabla 6. Entidades territoriales con comportamientos inusuales de mortalidad materna temprana respecto al promedio 2017-2020, Colombia, semana epidemiológica 13 de 2021

$\begin{array}{cccc}\text { Entidad territorial de residencia } & \text { Valor observado } & \text { Valor histórico } & \text { Poisson } \\ \text { Antioquia } & 11 & 7 & 0,045 \\ \text { Bogotá } & 9 & 5 & 0,036 \\ \text { Cauca } & 0 & 3 & 0,050 \\ \text { Guaviare } & 1 & 0 & 0,000 \\ \text { Huila } & 2 & 0 & 0,000 \\ \text { Nariño } & 1 & 5 & 0,034 \\ \text { Valle del Cauca } & 4 & 1 & 0,015 \\ \text { Cali } & 5 & 2 & 0,036\end{array}$

Fuente: Sivigila, Instituto Nacional de Salud, Colombia, 2017-2021

La información es notificada semanalmente por las entidades territoriales (ET) al Instituto Nacional de Salud (INS) a través del Sistema de vigilancia en salud pública (Sivigila). El número de casos, puede variar después de que se realizan unidades de análisis en las ET para el ajuste y la clasificación de caso en cada evento. 
Encuantoa las causas de muerte materna tempranael 21,7\% corresponde a causas directas y el $18,5 \%$ a causas indirectas. Las principales causas de muerte materna directa corresponden a trastorno hipertensivo asociado al embarazo con el 7,6 \% y la hemorragia obstétrica con el 5,4 \% (Tabla 7).

Tabla 7. Mortalidad materna temprana por tipo y causa principal agrupada, Colombia, semanas epidemiológicas 01 a 13 de 2021

\begin{tabular}{lrr} 
Causa agrupada & Casos & \multicolumn{1}{c}{} \\
DIRECTA & $\mathbf{2 0}$ & $\mathbf{2 1 , 7}$ \\
Trastorno hipertensivo asociado al embarazo & 7 & $\mathbf{7 , 6}$ \\
Hemorragia obstétrica & 5 & 5,4 \\
Sepsis relacionada con el embarazo & 4 & 4,3 \\
Evento tromboembolico como causa básica & 3 & 3,3 \\
Otras causas directas & 1 & 1,1 \\
INDIRECTA & $\mathbf{1 7}$ & $\mathbf{1 8 , 5}$ \\
Otras causas indirectas & 8 & 8,7 \\
Otras causas indirectas: Neumonía por COVID 19 & 4 & 4,3 \\
Sepsis no obstétrica: Neumonía & 4 & 4,3 \\
Sepsis no obstétrica & 1 & 1,1 \\
EN ESTUDIO & $\mathbf{5 5}$ & $\mathbf{5 9 , 8}$ \\
\hline
\end{tabular}

Fuente: Sivigila, Instituto Nacional de Salud, Colombia, 2021

Para el análisis de los comportamientos inusuales, por ser la mortalidad materna un evento de baja frecuencia, se usa la distribución de probabilidades

de Poisson por medio de la estimación de la probabilidad de ocurrencia del evento según su comportamiento medio entre el 2017 y 2020.

\section{Mortalidad perinatal y neonatal tardía}

A semana epidemiológica 13 de 2021, se han notificado 1935 casos de muerte perinatal y neonatal tardía (MPNT) de los cuales 28 corresponden a casos residentes en el exterior por lo que no se incluyen en el análisis. En esta semana se notificaron 123 casos, 92 corresponden a esta semana y 31 a notificaciones tardías.

En la semana epidemiológica analizada la razón preliminar nacional de mortalidad perinatal y neonatal tardía es de 12,8 muertes por cada 1000 nacidos vivos, en la tabla 8 se observa que en 20 entidades territoriales la razón es superior a la del país. Las 5 entidades territoriales con las razones más altas son Guainía $(49,1)$, Vichada $(29,9)$, Chocó $(28,2)$, Arauca $(23,8)$ y Barranquilla $(21,1)$.
Tabla 8. Número de casos y razón de mortalidad perinatal y neonatal tardía por entidad territorial de residencia, Colombia, semanas epidemiológicas 01 a 13 de 2021

\begin{tabular}{|c|c|c|}
\hline $\begin{array}{l}\text { Entidad territorial de } \\
\text { residencia }\end{array}$ & $\begin{array}{l}\text { Número de } \\
\text { casos }\end{array}$ & $\begin{array}{l}\text { Razón de Mortalidad perinatal y neonatal } \\
\text { tardía por } 1000 \text { nacidos vivos }\end{array}$ \\
\hline Guainía & 11 & 49,1 \\
\hline Vichada & 10 & 29,9 \\
\hline Chocó & 36 & 28,2 \\
\hline Arauca & 23 & 23,8 \\
\hline Barranquilla & 104 & 21,1 \\
\hline Córdoba & 118 & 20,2 \\
\hline Bolívar & 64 & 17,6 \\
\hline Putumayo & 19 & 17,5 \\
\hline La Guajira & 85 & 17,1 \\
\hline Amazonas & 4 & 16,7 \\
\hline Sucre & 53 & 16,5 \\
\hline Buenaventura & 17 & 16,5 \\
\hline Nariño & 57 & 14,6 \\
\hline Cauca & 60 & 14,5 \\
\hline Quindío & 19 & 14,5 \\
\hline Caquetá & 21 & 13,8 \\
\hline Caldas & 27 & 13,6 \\
\hline Cartagena & 51 & 13,5 \\
\hline Risaralda & 30 & 13,0 \\
\hline Antioquia & 224 & 12,9 \\
\hline Colombia & 1907 & 12,8 \\
\hline Boyacá & 41 & 12,7 \\
\hline Cundinamarca & 105 & 12,6 \\
\hline Cesar & 66 & 12,4 \\
\hline Atlántico & 54 & 12,3 \\
\hline Huila & 51 & 12,2 \\
\hline Casanare & 17 & 11,8 \\
\hline Tolima & 45 & 11,8 \\
\hline San Andrés y Providencia & 2 & 11,3 \\
\hline Valle del Cauca & 50 & 11,0 \\
\hline Cali & 64 & 11,0 \\
\hline Magdalena & 35 & 10,9 \\
\hline Santa Marta & 22 & 10,2 \\
\hline Meta & 36 & 9,7 \\
\hline Norte de Santander & 52 & 9,3 \\
\hline Bogotá & 184 & 9,2 \\
\hline Vaupés & 1 & 8,1 \\
\hline Santander & 47 & 7,2 \\
\hline Guaviare & 2 & 6,2 \\
\hline
\end{tabular}

Tema central

Situación $\mathrm{Nal}$

Mortalidad

Trazadores

Brotes

COVID-19

Tablas 
Según el momento de ocurrencia de la muerte la mayor proporción son muertes perinatales anteparto con 47,4 \% (904), seguido de neonatales tempranas con 28,2 \% (537), neonatales tardías con 14,6\% (279) y perinatales intraparto con $9,8 \%$ (187).

De acuerdo con las causas de muerte agrupadas se observó la mayor proporción en: otras causas de muerte $26,0 \%$, principalmente trastornos de origen perinatal y placentarios, seguido por prematuridad-inmaturidad $24,3 \%$ y asfixia y causas relacionadas $20,6 \%$ (Figura 6).

Figura 6. Proporción de causas de muertes perinatales y neonatales tardías por causas de muerte agrupadas, Colombia, semanas epidemiológicas 01 a 13 de 2021

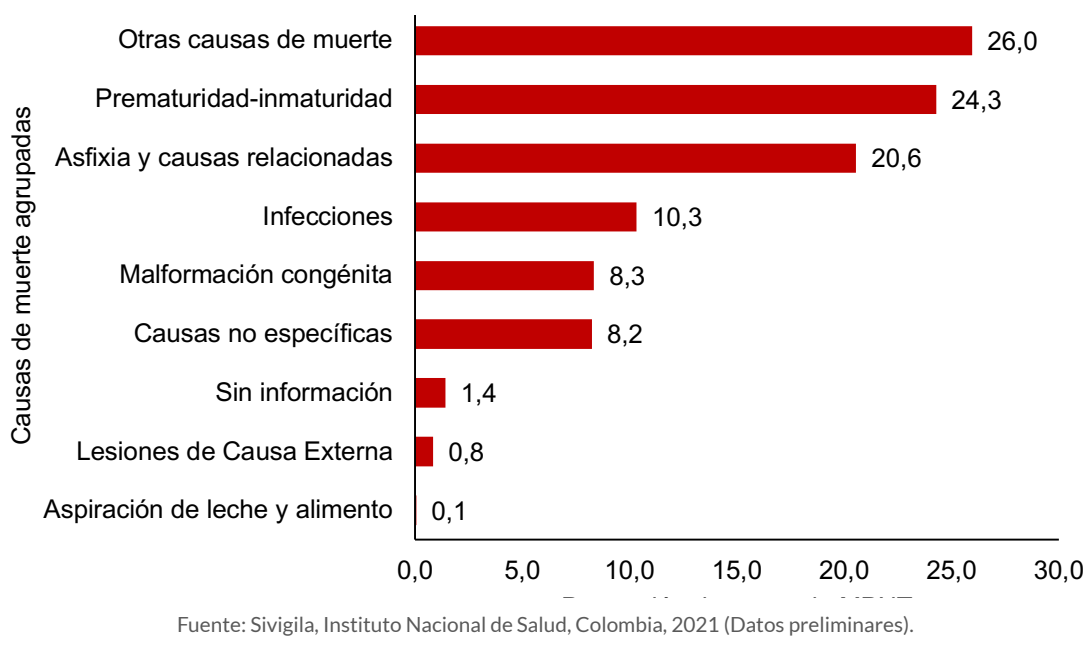

En lo observado en la semana de análisis, en comparación con lo notificado entre 2016 y 2021, se presenta un decremento en la notificación en Atlántico, Bogotá, Cesar, Cundinamarca, Risaralda, Santander y Tolima. Comportamiento que podría ser explicado por el subregistro en la notificación de las muertes a Sivigila, respecto al número de muertes certificadas en el Registro Único de Afiliados a la Protección Social-RUAF.
Dado que el evento tiene una alta frecuencia en notificación y se cuenta con una línea de base estable de más de cinco años, se realiza el análisis de los comportamientos inusuales a través del método: Morbidity and Mortality Weekly Report (MMWR) del Center for Disease Control and Prevention $(C D C)$. Este se basa en la comparación del comportamiento actual del evento en el último periodo epidemiológico (valor observado) con el resultado del promedio de 15 periodos históricos alrededor de ese periodo de evaluación, la ventana histórica de estimación contempla los cinco años previos al año de análisis (Coutin G, Borges J, Batista R, Feal P, Suárez B. Método para el análisis del comportamiento observado de enfermedades seleccionadas con relación al comportamiento histórico. Rev Cubana Hig Epidemiol. 2000;38(3):157-66).
Tema central

Situación Nal.

Mortalidad

Trazadores

Brotes

COVID-19

Tablas 


\section{EVENTOS TRAZADORES}

\section{Infección respiratoria aguda}

A nivel nacional se identifica disminución en la notificación para la morbilidad por infección respiratoria aguda (IRA) en los servicios de consulta externa y urgencias; se presenta incremento en las hospitalizaciones por IRAG en sala general y en unidad de cuidados intensivos e intermedios (tabla 9).

Tabla 9 . Notificación morbilidad por Infección Respiratoria Aguda por tipo de servicio en Colombia, semanas epidemiológicas 01 a 13, 2020 y 2021

\begin{tabular}{lrrrr} 
Tipo de servicio & 2020 a semana 13 & 2021 a semana 13 & \multicolumn{2}{c}{ Variación } \\
Consultas externas y urgencias & 1802309 & 963675 & $-46,5$ & Disminución \\
Hospitalizaciones en sala general & 53286 & 55117 & 3,4 & Aumento \\
Hospitalizaciones en UCl & 5673 & 18397 & 224,3 & Aumento \\
\hline \multicolumn{2}{c}{ Fuente: Sivigila, Instituto Nacional de Salud, Colombia, 2020 a 2021} & &
\end{tabular}

A semana epidemiológica 13 de 2021, en Colombia se han notificado 963675 consultas externas y urgencias por IRA, presentando disminución frente a lo notificado a la misma semana de los tres años anteriores con el 46,5\% comparado con 2020, 37,3\% frente a 2019 y $38,9 \%$ frente a 2018 . Teniendo en cuenta el comportamiento de notificación de los últimos siete años, se presenta disminución en 27 entidades territoriales; Córdoba y Santa Marta presentan incremento; Bolívar, Guainía, La Guajira, Magdalena, Risaralda, Santander, Sucre, Vaupés y Vichada no presentan comportamientos inusuales.

Por grupos de edad, los adultos de 20 a 39 años representan el 31,5 \% (303 700), seguido por el de 40 a 59 años con el 20,7 \% (199 192). La mayor proporción de consultas externas y de urgencias por IRA sobre el total de consultas por todas las causas se presenta en los niños de 1 año con el 8,3\% seguido de los menores de 1 año con el $8,0 \%$.

En el canal endémico las consultas externas y urgencias por IRA para las trece semanas del año se ubican por debajo del umbral estacional y a partir de la semana 02 por debajo del límite inferior (figura 7).
Figura 7. Canal endémico de consultas externas y urgencias por infección respiratoria aguda, Colombia, semanas epidemiológicas 01 a 13, entre 2014 y 2021

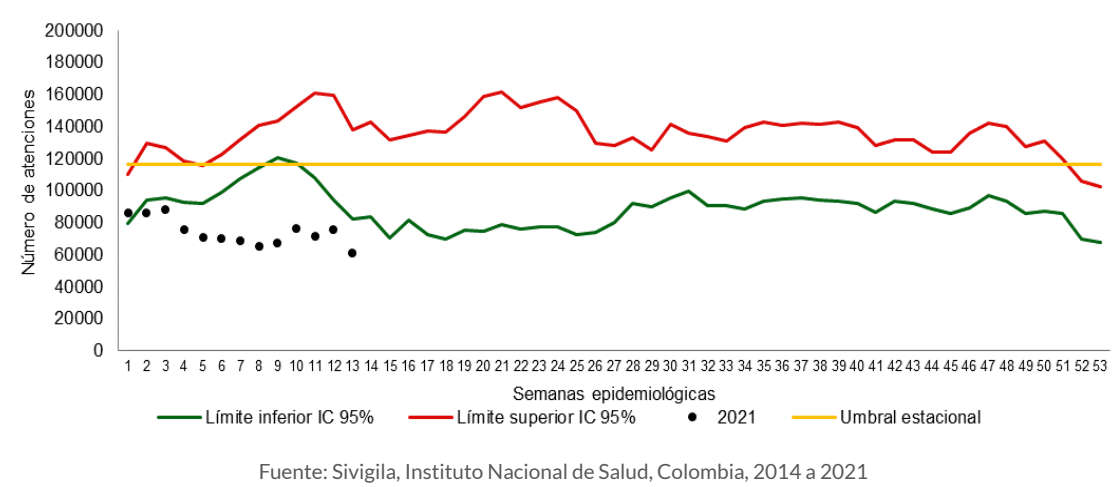

Se notificaron 55117 hospitalizaciones por IRAG en sala general, presentando incremento frente a lo notificado a la misma semana de los dos años anteriores con el 3,4 \% frente a 2020, 8,9\% comparado con 2019 y una disminución del $1,8 \%$ frente a 2018. Teniendo en cuenta el comportamiento de notificación de los últimos siete años, se presenta disminución en 18 entidades territoriales, entre las cuales resaltan Chocó, Vaupés, Caquetá, Quindío, Casanare, Atlántico, Arauca, Cauca y Guaviare; e incremento en 7 entidades territoriales resaltando Barranquilla, Amazonas, Santander y Tolima; no presentan comportamientos inusuales 13 entidades territoriales.

Por grupos de edad, los adultos de más de 60 años representan el 38,7 \% (21 313), seguido por los de 40 a 59 años con el 20,5 \% (11 318). La mayor proporción de hospitalizaciones en sala general por IRAG sobre el total de hospitalizaciones por todas las causas se presenta en los niños de 1 año con el $15,3 \%$, seguido por los niños de 2 a 4 años con el $12,4 \%$.

En el canal endémico durante las cuatro primeras semanas del año las hospitalizaciones por IRAG en sala general se ubicaron por encima del límite superior histórico esperado y el umbral estacional, para la semana 05 se observa un descenso por debajo del umbral estacional, sin variación para las semanas 06 a 08; de las semanas 09 a 12 se ubican en zona de éxito, por debajo del límite interior y para la semana 13 se observa un ligero aumento con respecto a la semana anterior ubicándose por encima del límite inferior (figura 8). 
Figura 8. Canal endémico de hospitalizaciones por infección respiratoria aguda grave en sala general, Colombia, semanas epidemiológicas 01 a 13, entre 2014 y 2021

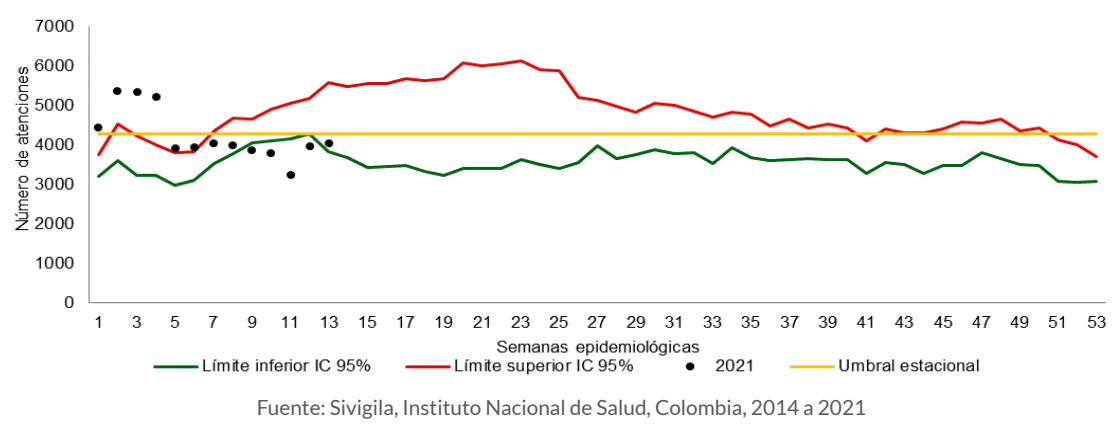

Se notificaron 18397 hospitalizaciones por IRAG en unidad de cuidados intensivos - $\mathrm{UCl}$ e intermedios, presentando incremento frente a lo notificado a la misma semana de los tres años anteriores con el 224,3\% frente a 2020, 289,4 \% comparado con 2019 y $283,9 \%$ frente a 2018 . Se presenta incremento en 29 entidades territoriales, entre las cuales resaltan Valle del Cauca, Caquetá, Antioquia, Santa Marta, Nariño, Barranquilla y Cundinamarca; San Andrés presenta disminución y no presentan comportamientos inusuales: Amazonas, Arauca, Chocó, Córdoba, Guainía, Guaviare, Putumayo y Vaupés.

Por grupos de edad, los adultos de más de 60 años representan el 55,4 \% (10 183), seguido por los de 40 a 59 años con el $26,1 \%$ (4 803) y los de 20 a 39 años con el 8,0 \% (1471). La mayor proporción de hospitalizaciones por IRAG en UCl e intermedios sobre el total de hospitalizaciones en $\mathrm{UCl}$ por todas las causas se presenta en el grupo de mayores de 60 años con el 28,0 $\%$, seguido por el de 40 a 59 años con el $27,4 \%$.

Hasta la semana 05 las hospitalizaciones por IRAG en UCI e intermedios superaron el promedio histórico y el límite superior de las semanas 01 a 53 de 2020 , adicionalmente se presenta un incremento progresivo hasta la tercera semana y un descenso por siete semanas; para las últimas tres semanas se observa incremento por encima del umbral estacional (figura 9).
Figura 9. Comportamiento de hospitalizaciones por infección respiratoria aguda grave en unidades de cuidados intensivos, Colombia, semanas epidemiológicas 01 a 13, entre 2020 y 2021

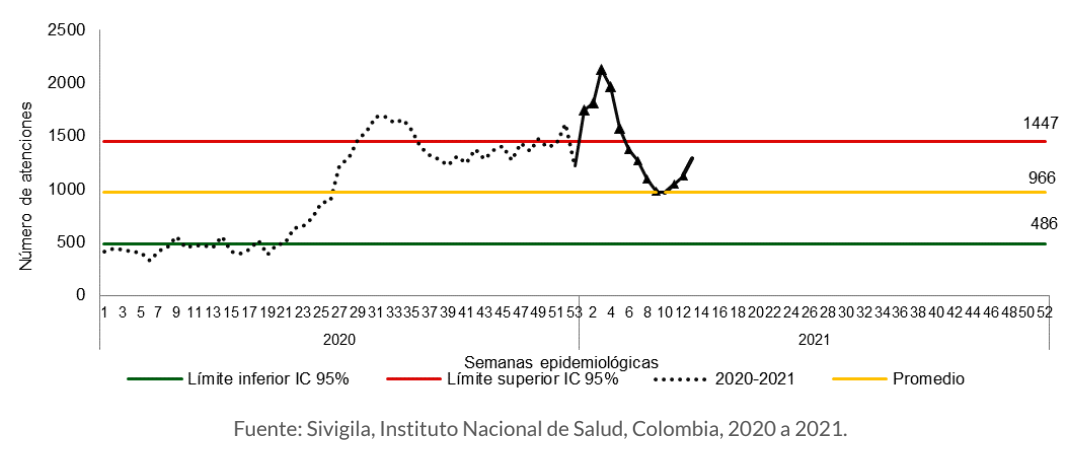

\section{METODOLOGÍA:}

Vigilancia de morbilidad por IRA: los canales endémicos para consultas externas y urgencias y hospitalizaciones en sala general se realizaron con la metodología de Bortman con los datos de la morbilidad por infección respiratoria aguda mediante el cálculo de la media geométrica de los años 2014 a 2020 y el intervalo de confianza.

Para las hospitalizaciones por IRAG en UCI e intermedio se construyó gráfico de control mediante el cálculo del promedio, la desviación estándar y el intervalo de confianza del año anterior.

\section{Dengue}

En la semana epidemiológica 13 de 2021 se notificaron 535 casos probables de dengue: 270 casos de esta semana y 265 casos de semanas anteriores. En el sistema hay 9235 casos, 4647 (50,3\%) sin signos de alarma, 4447 $(48,2 \%)$ con signos de alarma y $141(1,5 \%)$ de dengue grave.

En Colombia, los casos de dengue proceden de 32 departamentos, 4 distritos, 557 municipios. Las entidades territoriales de Cali, Valle del Cauca, Cartagena, Putumayo, Huila, Tolima, Antioquia, Barranquilla, Cundinamarca, Norte de Santander, Meta y Cesar, aportan el 72,1 \% (6 654) de los casos a nivel nacional (tabla 10). 
Tabla 10. Casos notificados de dengue por entidad territorial de procedencia y clasificación en Colombia, semanas epidemiológicas 01 a 13 de 2021

\begin{tabular}{|c|c|c|c|c|c|c|}
\hline \multirow{2}{*}{ Entidad territorial } & \multicolumn{2}{|l|}{ Dengue } & \multicolumn{4}{|c|}{ Dengue grave Total } \\
\hline & $n$ & $\%$ & $\mathrm{n}$ & $\%$ & $\mathrm{n}$ & $\%$ \\
\hline Cali & 2048 & 22.5 & 23 & 16.3 & 2071 & 22.4 \\
\hline Valle del Cauca & 727 & 8.0 & 7 & 5.0 & 734 & 7.9 \\
\hline Cartagena & 593 & 6.5 & 17 & 12.1 & 610 & 6.6 \\
\hline Putumayo & 500 & 5.5 & 1 & 0.7 & 501 & 5.4 \\
\hline Huila & 412 & 4.5 & 7 & 5.0 & 419 & 4.5 \\
\hline Tolima & 410 & 4.5 & 5 & 3.5 & 415 & 4.5 \\
\hline Antioquia & 361 & 4.0 & 5 & 3.5 & 366 & 4.0 \\
\hline Barranquilla & 342 & 3.8 & 6 & 4.3 & 348 & 3.8 \\
\hline Cundinamarca & 308 & 3.4 & 1 & 0.7 & 309 & 3.3 \\
\hline Norte de Santander & 291 & 3.2 & 6 & 4.3 & 297 & 3.2 \\
\hline Meta & 291 & 3.2 & 2 & 1.4 & 293 & 3.2 \\
\hline Cesar & 279 & 3.1 & 12 & 8.5 & 291 & 3.2 \\
\hline Atlántico & 262 & 2.9 & 4 & 2.8 & 266 & 2.9 \\
\hline Bolívar & 226 & 2.5 & 5 & 3.5 & 231 & 2.5 \\
\hline Santander & 215 & 2.4 & 3 & 2.1 & 218 & 2.4 \\
\hline Magdalena & 179 & 2.0 & 9 & 6.4 & 188 & 2.0 \\
\hline Sucre & 171 & 1.9 & 4 & 2.8 & 175 & 1.9 \\
\hline Córdoba & 164 & 1.8 & 2 & 1.4 & 166 & 1.8 \\
\hline Cauca & 151 & 1.7 & 1 & 0.7 & 152 & 1.6 \\
\hline Santa Marta & 132 & 1.5 & 4 & 2.8 & 136 & 1.5 \\
\hline Nariño & 123 & 1.4 & 4 & 2.8 & 127 & 1.4 \\
\hline Caquetá & 119 & 1.3 & 2 & 1.4 & 121 & 1.3 \\
\hline Casanare & 110 & 1.2 & 0 & 0.0 & 110 & 1.2 \\
\hline Boyacá & 103 & 1.1 & 1 & 0.7 & 104 & 1.1 \\
\hline Chocó & 98 & 1.1 & 1 & 0.7 & 99 & 1.1 \\
\hline Amazonas & 78 & 0.9 & 0 & 0.0 & 78 & 0.8 \\
\hline Quindío & 75 & 0.8 & 2 & 1.4 & 77 & 0.8 \\
\hline Buenaventura & 68 & 0.7 & 1 & 0.7 & 69 & 0.7 \\
\hline Arauca & 50 & 0.5 & 0 & 0.0 & 50 & 0.5 \\
\hline Risaralda & 49 & 0.5 & 0 & 0.0 & 49 & 0.5 \\
\hline Caldas & 46 & 0.5 & 0 & 0.0 & 46 & 0.5 \\
\hline La Guajira & 33 & 0.4 & 4 & 2.8 & 37 & 0.4 \\
\hline Archipiélago de San Andrés & 26 & 0.3 & 0 & 0.0 & 26 & 0.3 \\
\hline Guaviare & 20 & 0.2 & 0 & 0.0 & 20 & 0.2 \\
\hline Exterior & 18 & 0.2 & 2 & 1.4 & 20 & 0.2 \\
\hline Vichada & 10 & 0.1 & 0 & 0.0 & 10 & 0.1 \\
\hline Guainía & 6 & 0.1 & 0 & 0.0 & 6 & 0.1 \\
\hline Total & 9094 & 100 & 141 & 100 & 9235 & 100 \\
\hline
\end{tabular}

Fuente: Sivigila, Instituto Nacional de Salud, Colombia, 2021
El 62,1\% (5 735) de los casos de dengue se reportó en 35 municipios, con mayor frecuencia en: Cali, con 22,4 \% (2 071); Cartagena, con el 6,6 \% (610); Barranquilla, con 3,8 \% (348); Orito, con el 2,1 \% (190); Cúcuta, con el 1,9\% (174); Santa Marta, con el 1,5\% (136); Neiva, con el 1,4\% (127); Ibagué, con 1,3\% (116); Villavicencio (113) y Aguachica (112), con el 1,2 \% cada uno; Yumbo (103) y Villagarzón (97), con el 1,1 $\%$ cada uno.

Se hospitalizó el 75,6\% (3 362) de los casos de dengue con signos de alarma y el 90,1\% (127) de dengue grave; las entidades notificadoras que hospitalizaron menos del 60,0 \% de los casos de dengue con signos de alarma fueron: Valle del Cauca, Buenaventura, Caldas, Cauca, Bolívar, Chocó y Vichada; y las entidades notificadoras que no hospitalizaron el $100 \%$ de los casos de dengue grave fueron: Cali, Santa Marta, Cartagena, Magdalena, Antioquia, Santander, La Guajira, Cundinamarca y Quindío.

A semana 13 se ha confirmado el 43,2\% (1920) de los casos de dengue con signos de alarma, las entidades que han confirmado el 100,0 \% de los casos notificados son Buenaventura y Vichada. Por otro lado, se ha confirmado el $53,9 \%$ (76) de los casos de dengue grave y las entidades con el 100,0 \% de los casos confirmados son Atlántico, Barranquilla, Buenaventura, Caquetá, Chocó, Córdoba y La Guajira.

La incidencia nacional de dengue es de 29,9 casos por cada 100000 habitantes en riesgo. En las entidades de Amazonas, Putumayo, Cali, Cartagena, Cundinamarca y Archipiélago de San Andrés y Providencia se estiman tasas de incidencia superiores a 53,0 casos por 100000 habitantes (figura 10). 
Figura 10. Incidencia de dengue por entidad territorial de procedencia en Colombia, semana epidemiológica 13 de 2021

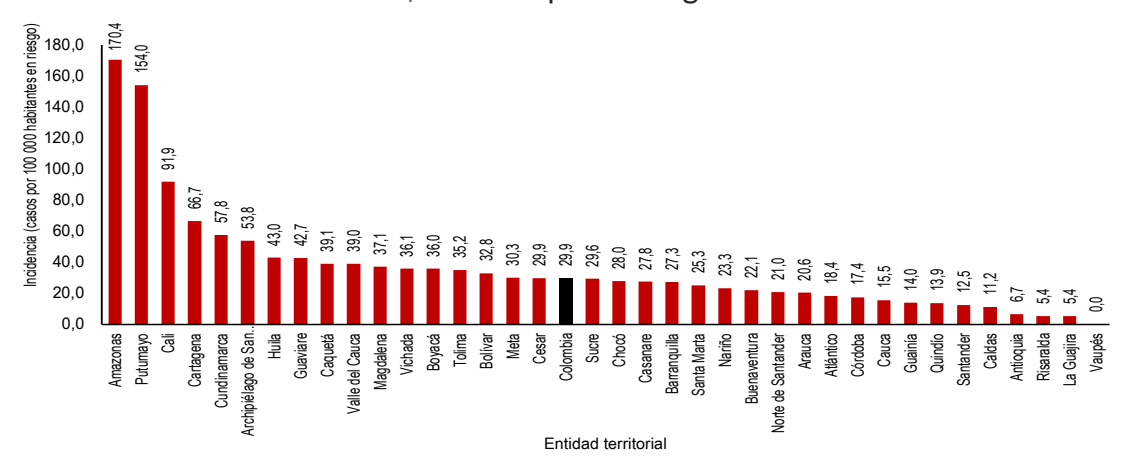

Fuente: Sivigila, Instituto Nacional de Salud, Colombia, 2021

A semana epidemiológica 13 de 2021, el evento a nivel nacional presentó un comportamiento dentro de lo esperado, comparado con su comportamiento histórico (figura 11).

Figura 11. Canal endémico nacional de dengue en Colombia, semana epidemiológica 13, 2021

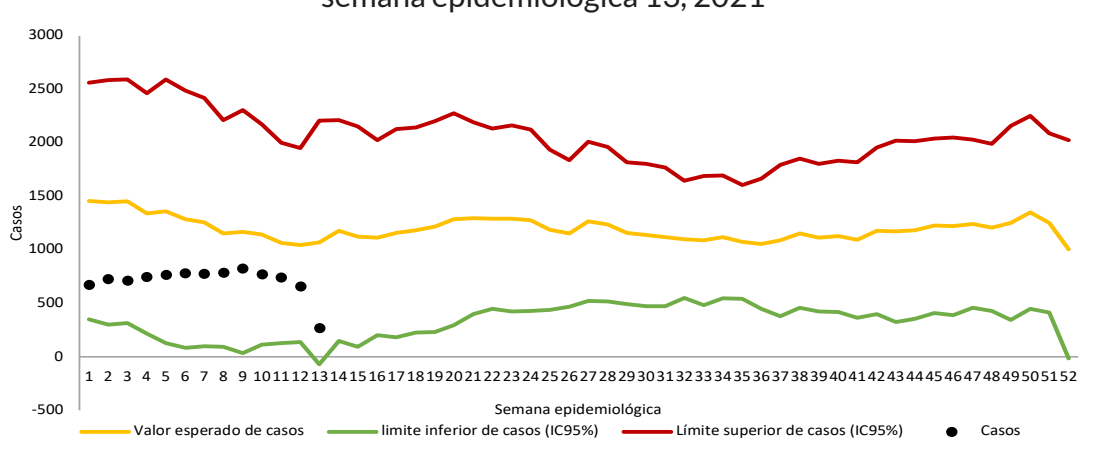

Fuente: Sivigila, Instituto Nacional de Salud, Colombia, 2021

De acuerdo con la situación epidemiológica según canal endémico, 19 entidades territoriales se encuentran dentro de lo esperado, 14 entidades se encuentran en situación de alerta y 4 entidades con presentación de casos por encima del valor esperado, comparado con el comportamiento histórico (tabla 11).
Tabla 11. Comparación de los casos notificados de dengue con su comportamiento histórico, por entidad territorial de procedencia en Colombia, semanas epidemiológicas 10 a 12 de 2021

\begin{tabular}{|c|c|c|c|}
\hline $\begin{array}{l}\text { Comportamiento } \\
\text { epidemiológico }\end{array}$ & \multicolumn{3}{|c|}{ Entidad territorial } \\
\hline $\begin{array}{l}\text { Dentro del número } \\
\text { esperado de casos }\end{array}$ & $\begin{array}{l}\text { Antioquia } \\
\text { Casanare } \\
\text { Guainía } \\
\text { La Guajira } \\
\text { Quindío } \\
\text { Sucre } \\
\text { Vichada } \\
\end{array}$ & $\begin{array}{l}\text { Arauca } \\
\text { Córdoba } \\
\text { Guaviare } \\
\text { Meta } \\
\text { Risaralda } \\
\text { Tolima }\end{array}$ & $\begin{array}{l}\text { Caldas } \\
\text { Cundinamarca } \\
\text { Huila } \\
\text { Norte de Santander } \\
\text { Santander } \\
\text { Vaupés } \\
\end{array}$ \\
\hline Situación de alerta & $\begin{array}{l}\text { Amazonas } \\
\text { Boyacá } \\
\text { Cauca } \\
\text { Nariño } \\
\text { San Andrés. }\end{array}$ & $\begin{array}{l}\text { Atlántico } \\
\text { Cali } \\
\text { Cesar } \\
\text { Putumayo } \\
\text { Cia y Santa Catalina }\end{array}$ & $\begin{array}{l}\text { Bolívar } \\
\text { Caquetá } \\
\text { Chocó } \\
\text { Santa Marta } \\
\text { Valle del Cauca }\end{array}$ \\
\hline $\begin{array}{l}\text { Por encima del número } \\
\text { esperado de casos }\end{array}$ & $\begin{array}{l}\text { Barranquilla } \\
\text { Magdalena }\end{array}$ & Buenaventura & Cartagena \\
\hline
\end{tabular}

Durante el 2021 se han notificado 34 muertes probables por dengue, de las cuales 6 han sido confirmadas, procedentes de: Cartagena, con 4 casos; Barranquilla y Córdoba, con 1 caso cada uno. Se han descartado 5 casos y se encuentran en estudio 23 muertes procedentes de: Magdalena, con 4 casos; Cali, Cesar y Valle del Cauca, con 3 casos cada uno; Santa Marta, con 2 casos; Atlántico, Barranquilla, Bolívar, Archipiélago de San Andrés y Providencia, Huila, Nariño, Quindío y Exterior, con 1 caso cada uno.

\section{Metodología}

Se realizó un informe descriptivo de los casos notificados durante la semana epidemiológica 13 de 2021, teniendo en cuenta las variables de tiempo, persona y lugar contenidas en la ficha de datos básicos y complementarios del evento de dengue, dengue grave y mortalidad por dengue (Código INS 210, 220 y 580). Los indicadores se presentan en distribuciones de frecuencias en figuras y tablas.

El canal endémico nacional y el análisis de comportamiento epidemiológico por entidad territorial se realizó con la metodología de medias geométricas (Marcelo Bortman), estableciendo los siguientes límites de control: por debajo de lo esperado, número de casos menor al límite inferior IC95\%; dentro de lo esperado, número de casos entre el límite inferior y la media geométrica IC $95 \%$; en alerta, número de casos entre la media geométrica y el límite superior IC $95 \%$, e incremento por encima de lo esperado, número de casos por encima del límite superior IC95\%.

En el análisis del comportamiento de dengue por canal endémico no se tiene en cuenta la semana epidemiológica actual dado que, el periodo de incubación del virus de dengue es de 3 a 14 días, por lo tanto, los casos de esta semana se reflejarán plenamente en la siguiente semana. 


\section{Malaria}

\section{Análisis epidemiológico nacional}

Según el análisis del último periodo epidemiológico, el país se encuentra en situación de seguridad para malaria, como lo muestra el canal endémico (Figura 12).

Figura 12. Canal endémico de malaria, Colombia, semana epidemiológica 13, 2021

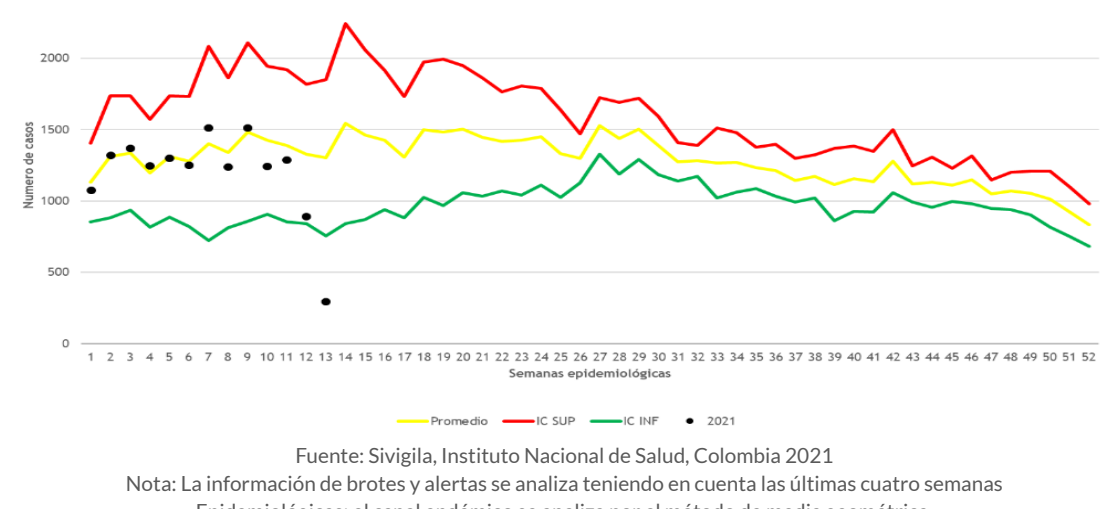
Epidemiológicas: el canal endémico se analiza por el método de media geométrica.

En la semana epidemiológica 13 se notificaron 912 casos de malaria, teniendo un acumulado de 15707 casos, de los cuales 15384 son de malaria no complicada y 323 de malaria complicada. Predomina la infección por Plasmodium vivax (P. vivax) con 49,8 \% (7 822), seguido de Plasmodium falciparum (P. falciparum) con 49,5\% (7777) e infección mixta con 0,7\% (108).

\section{Malaria no complicada}

Por procedencia, Chocó (29,7 \%), Nariño (23,6 \%), Córdoba (14,0 \%), Antioquia (10,3\%) y Guainía $(4,7)$ aportan el $82,3 \%$ de los casos de malaria no complicada.
Tabla 12. Casos notificados de malaria no complicada por entidad territorial de procedencia. Colombia, semanas epidemiológicas 01 a 13 de 2021

\begin{tabular}{|c|c|c|c|c|c|c|}
\hline Entidad territorial & Infección mixta & P. falciparum & P. malarie & P. vivax & $\mathrm{n}$ & $\%$ \\
\hline Chocó & 40 & 2448 & 0 & 2087 & 4575 & 29,70 \\
\hline Nariño & 17 & 3233 & 0 & 382 & 3632 & 23,60 \\
\hline Córdoba & 6 & 413 & 0 & 1735 & 2154 & 14,00 \\
\hline Antioquia & 7 & 511 & 0 & 1060 & 1578 & 10,30 \\
\hline Guainía & 1 & 50 & 0 & 675 & 726 & 4,70 \\
\hline Cauca & 1 & 520 & 0 & 8 & 529 & 3,40 \\
\hline Vichada & 7 & 85 & 0 & 256 & 348 & 2,30 \\
\hline Norte de Santander & 0 & 0 & 0 & 343 & 343 & 2,20 \\
\hline Buenaventura & 2 & 255 & 0 & 47 & 304 & 2,00 \\
\hline Guaviare & 2 & 45 & 0 & 219 & 266 & 1,70 \\
\hline Bolívar & 12 & 19 & 0 & 190 & 221 & 1,40 \\
\hline Risaralda & 1 & 8 & 0 & 209 & 218 & 1,40 \\
\hline Amazonas & 2 & 12 & 0 & 147 & 161 & 1,00 \\
\hline Meta & 0 & 21 & 0 & 133 & 154 & 1,00 \\
\hline Casanare & 0 & 0 & 0 & 53 & 53 & 0,30 \\
\hline Exterior & 0 & 10 & 0 & 22 & 32 & 0,21 \\
\hline Vaupés & 0 & 0 & 0 & 16 & 16 & 0,10 \\
\hline Cali & 0 & 9 & 0 & 4 & 13 & 0,10 \\
\hline Desconocido & 0 & 2 & 0 & 7 & 9 & 0,06 \\
\hline Sucre & 0 & 1 & 0 & 6 & 7 & 0,00 \\
\hline Cesar & 1 & 1 & 0 & 5 & 7 & 0,00 \\
\hline Putumayo & 0 & 0 & 0 & 6 & 6 & 0,00 \\
\hline Valle del Cauca & 0 & 5 & 0 & 1 & 6 & 0,00 \\
\hline La Guajira & 0 & 0 & 0 & 6 & 6 & 0,00 \\
\hline Santander & 1 & 1 & 0 & 3 & 5 & 0,00 \\
\hline Arauca & 0 & 0 & 0 & 5 & 5 & 0,00 \\
\hline Cartagena & 0 & 0 & 0 & 4 & 4 & 0,00 \\
\hline Huila & 0 & 0 & 0 & 2 & 2 & 0,00 \\
\hline Barranquilla & 1 & 0 & 0 & 0 & 1 & 0,00 \\
\hline Caquetá & 0 & 0 & 0 & 1 & 1 & 0,00 \\
\hline Atlántico & 0 & 0 & 0 & 1 & 1 & 0,00 \\
\hline Total & 101 & 7650 & 0 & 7633 & 15384 & 100 \\
\hline
\end{tabular}

Nota: no se presentaron casos de otros departamentos o distritos

\section{Malaria Complicada}

Se notificaron 323 casos de malaria complicada, que proceden de 20 entidades territoriales y un caso procedente del exterior. Nariño,Antioquia, Chocó, Córdoba, Meta y Vichada notifican el 73,0 \% de los casos.

De los casos de malaria complicada el $58,2 \%$ (188) corresponde a hombres. El 30,6 \% (99) se presenta en personas de 15 a 24 años y el 19,5\% (63) ocurre en indígenas. Por lugar de procedencia, el 42,1\% (136) proviene de rural disperso. Por régimen de afiliación, el 68,4 \% (221) pertenece al régimen subsidiado. 
De los casos de malaria complicada causados por P. vivax o P. falciparum, el 76,8 \% (248) presentó complicaciones hematológicas, el 17,0 \% (55) complicaciones hepáticas, el 6,5\% (21) complicaciones renales, el 3,1 \% (10) complicaciones pulmonares y el 2,5\% (8) malaria cerebral (Figura 13).

Figura 13. Tipo de complicaciones de malaria, Colombia, semanas epidemiológicas 01 a 13 de 2021

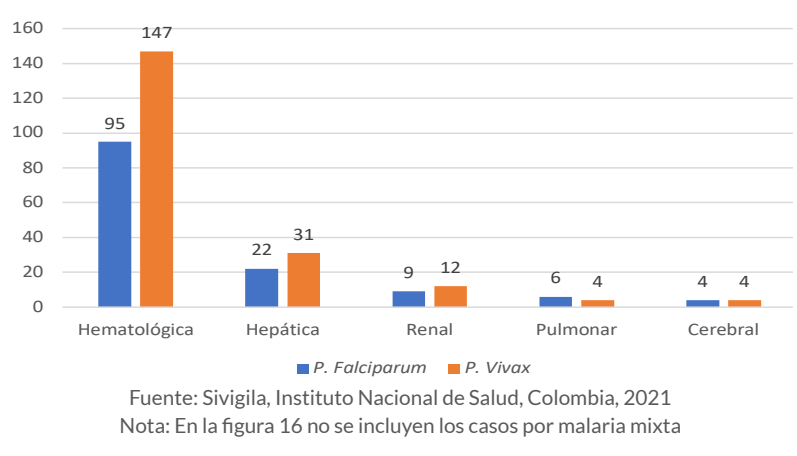

\section{Comportamientos inusuales}

A semana epidemiológica 13, tres departamentos están por encima del número esperado de casos y cuatro departamentos están en situación de alerta para malaria (Tabla 13).

Tabla 13. Distribución de entidades territoriales según el comportamiento epidemiológico de malaria, Colombia, semana epidemiológica 13 de 2021

\begin{tabular}{llll}
\hline Comportamiento epidemiológico & Departamentos & & \\
\cline { 4 - 4 } Menor al comportamiento histórico & Amazonas & Vaupés & Caquetá \\
\hline En el comportamiento histórico & La Guajira & Arauca & Atlántico \\
& Caquetá & Quindío & Caldas \\
& Huila & Sucre & Magdalena \\
& Cesar & Chocó & Santander \\
& Valle del Cauca & Vichada & Bolívar \\
& & Putumayo & Casanare \\
\hline Situación de alerta & Guainía & Nariño & Guaviare \\
& & Cauca \\
\hline Mayor al comportamiento histórico & Meta & Córdoba & Risaralda \\
\hline \multicolumn{2}{r}{ Fuente: Sivigila, Instituto Nacional de Salud, Colombia 2021 } &
\end{tabular}

Fuente: Sivigila, Instituto Nacional de Salud, Colombia 2021
Nota: La información de brotes yalertas se analiza teniendo en cuenta las últimas cuatro semanas epidemiológicas
A semana epidemiológica 13, 15 municipios se encuentran en situación de brote; los que más reportan casos son: El Charco (Nariño), Bojayá (Chocó), Vigía del Fuerte (Antioquia) y Mosquera y Maguí (Nariño) (Tabla 14).

Tabla 14. Municipios en situación de brote por malaria a semana epidemiológica 13 en Colombia, 2021

\begin{tabular}{lcccc} 
Departamento & Municipio & Acumulado & Esperado & Observado \\
\cline { 2 - 5 } Nariño & El Charco & 510 & 115 & 221 \\
Chocó & Bojayá & 417 & 82 & 161 \\
Antioquia & Vigía Del Fuerte & 385 & 84 & 157 \\
Nariño & Maguí & 354 & 100 & 155 \\
Nariño & Mosquera & 347 & 39 & 129 \\
Risaralda & Pueblo Rico & 221 & 29 & 62 \\
Chocó & Carmen del Darién & 163 & 26 & 45 \\
Antioquia & Murindó & 155 & 20 & 42 \\
Nariño & La Tola & 132 & 25 & 49 \\
Córdoba & Montería & 86 & 3 & 36 \\
Meta & Mapiripán & 74 & 2 & 23 \\
Chocó & Litoral del Bajo & 51 & 8 & 18 \\
Meta & San Juan & 44 & 5 & 8 \\
Meta & Puerto Gaitán & 23 & 3 & 12 \\
Nariño & Puerto Rico & 22 & 4 & 6 \\
& Policarpa & 22 &
\end{tabular}

\section{Comportamientos inusuales (municipios IREM)}

La Iniciativa Regional para la Eliminación de la Malaria (IREM) se implementa en doce municipios del pacífico colombiano, con el objetivo de reducir y eliminar la transmisión de la malaria. Busca fortalecer la red de diagnóstico para propender por un diagnóstico y tratamiento oportuno de casos de malaria, plantea la vigilancia en salud pública como intervención en salud pública y busca cortar cadenas de transmisión.

A semana epidemiológica 13, de los 12 municipios en los que se implementa la IREM, según el análisis de comportamientos inusuales, se encuentra en incremento el distrito de Buenaventura y en situación de seguridad según el análisis de canales endémicos. Se encuentran en decremento: Acandí, Atrato, Bagadó, Lloró, Medio Atrato, Quibdó, Rioquito y Unguía, ninguno se encuentra en situación de brote (Tabla 15). 
Tabla 15. Comportamientos inusuales, municipios Iniciativa Regional para la Eliminación de la Malaria, semana epidemiológica 12, Colombia, 2021

\begin{tabular}{lllllll}
\hline Departamento & Municipios & Acumulado & Esperado & Observado & $\begin{array}{c}\text { Comportamiento } \\
\text { inusual }\end{array}$ & $\begin{array}{c}\text { Situación } \\
\text { epidemiológica }\end{array}$ \\
\cline { 2 - 7 } & Acandí & 8 & 9 & 0 & Disminución & Éxito \\
& Atrato & 97 & 69 & 34 & Disminución & Éxito \\
& Bagadó & 181 & 134 & 55 & Disminución & Seguridad \\
& Juradó & 6 & 4 & 3 & Ninguno & Seguridad \\
Chocó & Lloró & 115 & 148 & 42 & Disminución & Éxito \\
& Medio Atrato & 142 & 91 & 53 & Disminución & Seguridad \\
& Quibdó & 1001 & 658 & 252 & Disminución & Éxito \\
& Rioquito & 78 & 73 & 26 & Disminución & Éxito \\
& Ríosucio & 58 & 10 & 15 & Ninguno & Seguridad \\
\hline Buenaventura & Unguía & 5 & 8 & 3 & Disminución & Seguridad \\
\hline Nariño & Buenaventura & 311 & 89 & 117 & Aumento & Seguridad \\
\hline & Tumaco & 509 & 242 & 172 & Ninguno & Seguridad \\
\hline
\end{tabular}

Fuente: Sivigila, Instituto Nacional de Salud, Colombia 2021

Nota: La información de brotes y alertas se analiza teniendo en cuenta las últimas cuatro semanas epidemiológicas

\section{Metodología}

Se realizó un análisis descriptivo con corte a semana epidemiológica 13 de 2021, con información que incluye: descripción de los casos en tiempo, lugar y persona, análisis de tendencia, comportamientos inusuales, descripción y análisis de indicadores para la vigilancia.

\section{BROTES Y SITUACIONES DE EMERGENCIA EN SALUD PÚBLICA}

\section{ALERTAS NACIONALES}

\section{Brote de intoxicación por sustancias químicas. población es- pecial. Puerto Salgar, Cundinamarca. 9 de abril de 2021}

El 8 de abril se notificó brote de intoxicación por sustancias químicas en batallón de Puerto Salgar, Cundinamarca. De acuerdo con la investigación epidemiológica de campo se identificaron 34 expuestos de los cuales 14 presentaron intoxicación por sustancias químicas. La edad promedio de los pacientes fue de 21 años. Todos recibieron manejo intrahospitalario. 4 se encuentran en $\mathrm{UCl}$. No se reportan fallecidos. El cuadro clínico identificado fue alteración del estado de consciencia, dolor abdominal, sialorrea, episodios eméticos no controlados, signos de dificultad respiratoria consistentes en estertores diseminados en ambos campos pulmonares, polipnea y taquicardia. La probable fuente de contaminación de los alimentos preparados es atribuida a un insecticida similar a la cipermetrina por mal almacenamiento. Se tomaron muestras de los alimentos aparentemente consumidos (4 muestras), así mismo muestras de suero y orina de los afectados. Situación en seguimiento.

Fuente: Secretaría de Salud Departamental, Cundinamarca, Abril 2021

\section{Brote de varicela. Población privada de la libertad. Bogotá. 05 de abril de 2021}

Se recibió información de un caso, hombre de 23 años, quien inició sintomatología el 25 de marzo consistente en fiebre, prurito, erupción cutánea pápulovesicular en tronco y cuello. Consultó al servicio de salud del establecimiento penitenciario el 28 de marzo. De acuerdo con la investigación epidemiológica de campo, realizada por la entidad territorial se identificaron 35 personas expuestas con una tasa de ataque del 2,9\%. Se establecieron medidas de control (restricción de 
visitas, y movilidad al interior de los patios, además de aislamiento de positivos) y se realizarán búsquedas activas con frecuencia diaria. Así mismo, se reforzarán las medidas de bioseguridad y desinfección. Situación en seguimiento.

Fuente: Secretaría de Salud Distrital. Bogotá, Abril 2021

\section{ALERTAS INTERNACIONALES}

\section{Actualización epidemiológica semanal de COVID-19. Fecha de Publicación 6 de abril 2021. Organización Mundial de la Salud (OMS)}

A nivel mundial los casos de COVID-19 han incrementado por sexta semana consecutiva con 4 millones de nuevos casos en la última semana. El número de muertes incrementó un $11 \%$ comparado con la semana anterior con más de 71000 nuevas muertes reportadas. EI mayor incremento se observó en el Sudeste Asiático (más notable en India) y la región del Pacifico Occidental. Todas las regiones excepto África reportado incremento en el número de muertes siendo el Sudeste Asiático el que presento el mayor aumento con $46 \%$.

El mayor número de caso se reportó en la India (513 885 nuevos casos; incremento del $38 \%$ ), Brasil (505 668 nuevos casos; con $5 \%$ de disminución), Estados Unidos de América (444 756 casos nuevos; incremento del $5 \%$ ), Turquía (256 937 casos nuevos; incremento del 43 \%) y Francia (244 607 nuevos casos, con disminución del 4 \%).

Región de África. La región reportó alrededor de 59000 casos nuevos y más de 1000 nuevas muertes la última semana con una disminución del $6 \%$ y $20 \%$ respectivamente comparado con la semana pasada. El mayor número de casos nuevos se notificó en Etiopía (12,6 casos nuevos por 100000 habitantes, un aumento del $10 \%$ ), Kenia (16,3 casos nuevos por 100000 una disminución del $5 \%$ ) y Sur África (11,9 casos nuevos por 100000 habitantes). El mayor número de nuevas muertes se registró en Sudáfrica (0,5 muertes por 100000 habitantes, una disminución del $46 \%$ ), Etiopia ( $<0,1$ nuevas muertes por 100000 , un aumento del $11 \%$ ) y Kenia (0,2 nuevas muertes por 100000 habitantes)

Región de las Américas. La región ha reportado más de 1.3 millones de casos nuevos y más de 37000 nuevas muertes con un incremento del $3 \%$ y $11 \%$ respectivamente, comparado con la semana anterior. En general se ha presentado una tendencia creciente en los casos notificados en las últimas seis semanas. El mayor número de casos nuevos se notificó en Brasil (237,9 casos nuevos por 100 000; una disminución del $5 \%$ ), los Estados Unidos de América (134,4 casos nuevos por 100 000; un $5 \%$ aumento) y Argentina (181,7 casos nuevos por 100 000; un aumento del $46 \%$ ). El mayor número de nuevas muertes se registró en Brasil (9,9 nuevas muertes por 100000 ; un aumento del 26 \%), los Estados Unidos de América (2,3 nuevas muertes por 100 000; un aumento del $8 \%$ ) y México (2,3 nuevas muertes por 100 000; una disminución del $18 \%$ ).

La Región del Mediterráneo Oriental notificó algo menos de 297000 nuevos casos y más de 3700 nuevas muertes, un aumento del $10 \%$ y del $7 \%$, respectivamente, en comparación con la semana anterior. Tanto el número de casos y muertes han presentado una trayectoria ascendente con nuevos casos en las últimas ocho semanas. El mayor número de casos nuevos se notificó en República Islámica de Irán (87,5 casos nuevos por 100 000; un aumento del $38 \%$ ), Jordania (438,5 casos nuevos por 100000 ; un $19 \%$ disminución) e Irak (102,0 casos nuevos por 100 000; un aumento del $9 \%$ ). El mayor número de nuevas muertes se registró en la República Islámica de Irán (0,8 nuevas muertes por 100 000; un aumento del $18 \%$ ), Jordania (6,4 nuevas muertes por 100 000; un $4 \%$ disminución) y Pakistán (0,2 nuevas muertes por 100 000; un aumento del $50 \%$ ).

Región Europea. Después de reportar un incremento progresivo de casos en las últimas cinco semanas, el número de casos nuevos reportados la semana pasada es similar a la reportada previamente con cerca de 6.2 millones de casos nuevos. El número de muertes continúa aumentando en las últimas cuatro semanas con 24000 nuevas muer- 
tes reportadas. El mayor número de casos nuevos se notificó en Turquía (315,3 casos nuevos por 100 000; un aumento del $43 \%$ ), Francia (374,7 casos nuevos por 100 000; una disminución del $4 \%$ ) y Polonia (495,6 casos nuevos por 100 000; una disminución del $3 \%$ ). El mayor número de nuevas muertes se registró en Italia (5,1 nuevas muertes por 100 000; un aumento del $2 \%$ ), la Polonia (8,1 nuevas muertes por 100 000; un aumento del 18 \%) y la Federación de Rusia (1,8 nuevas muertes por 100 000; una disminución del $3 \%$ ).

Región de Asia Sudoriental. En la última semana, la región del Sudeste Asiático notificó cerca de 592000 nuevos casos y 4300 nuevas muertes, un aumento del $36 \%$ y del $46 \%$, respectivamente, en comparación con la semana anterior. La tasa de incidencia estuvo aumentando marcadamente en el mes de marzo, con un marcado incremento en las últimas tres semanas. El número de fallecidos continúa aumentando en las últimas tres semanas con un fuerte incremento en la última semana. La India contribuye con el $87 \%$ de los casos nuevos y el $71 \%$ de las nuevas muertes en la región la semana pasada. El mayor número de casos se reportó en la India (37,2 casos nuevos por 100 000; un aumento del $38 \%$ ), Bangladesh (23,4 casos nuevos por 100 000; un aumento del $67 \%$ ) e Indonesia (13 casos nuevos por 100 000; un 2 $\%$ de disminución). El mayor número de nuevas muertes se registró en India (0,2 nuevas muertes por 100000; un aumento del $71 \%$ ), Indonesia (0,3 nuevas muertes por 100 000; una disminución del $4 \%$ ) y Bangladesh. (0,2 nuevas muertes. Por 100 000; un aumento del $71 \%)$.

Región del Pacífico Occidental. La semana anterior se notificaron más de 84000 nuevos casos y poco más de 500 nuevas muertes, un aumento del $32 \%$ y del $7 \%$, respectivamente, en comparación con la semana anterior. La Región ha informado de un fuerte aumento en el número de nuevos casos durante las últimas tres semanas. El mayor número de casos nuevos se notificó en Filipinas ( 51,5 casos nuevos por 100 000; un aumento del $43 \%$ ), Japón (8,9 casos nuevos por 100 000; un aumento del $28 \%$ ), y Malasia (27,6 casos nuevos por 100000 ; una disminución del $4 \%$ ). El mayor número de nuevas muertes se registró en Filipinas (0,2 nuevas muertes por 100 000; un aumento del $40 \%$ ), Japón (0,2 nuevas muertes por 100 000; una disminución del $13 \%$ y el República de Corea (<0,1 nuevas muertes por 100 000; una disminución del $4 \%$ ).

Fuente: Organización Mundial de la Salud (OMS). Actualización epidemiológica semanal de COVID-19. Fecha de Publicación 30 de marzo de 2021. Fecha de consulta 08 de abril de 2021. Disponible en: https:// www.who.int/publications/m/item/weekly-epidemiological-update-on-covid-19---31-march-2021

Virus del Ébola. Boletín de brotes y emergencias semana 13 Epidemiológico. Fecha de publicación 28 de marzo de 2021 - Organización Mundial de la Salud (OMS)

\section{Guinea}

Un nuevo caso la enfermedad del virus del Ébola (EVE) fue reportado el 25 de marzo de 2021 en Soulouta. Al 4 de abril de 2021 se reportaron un total de 23 casos incluyendo 16 casos confirmados y siete casos probables con 9 recuperados y 12 fallecidos (letalidad 52,2\%). La subprefectura de Soulouta permanece activa (dos casos confirmados y tres probables reportados en los últimos 21 días) mientras que Samoe y Nzerekore-Centro están en alerta. El número de trabajadores de salud confirmados son cinco. La mayoría de los casos confirmados son mujeres (13/23: 60,9\%) y el grupo de edad más afectado son aquellos mayores de 40 años.

Al 4 de abril un total de 43 (29\%) de 149 contactos han recibido seguimiento en Nzerekore. Un total de 101 contactos han sido vacunados. Se han presentado 19 alertas en Nzerekore al 4 de abril de 2021, de las cuales 11 (58\%) fueron investigadas dentro de las 24 horas, de estas, ocho fueron verificadas y de estas a seis se les había tomado muestra.

La respuesta en salud publica incluye el inicio de la vacunación con un total acumulado 4565 personas vacunadas incluidas 288 contactos de alto riesgo y 3835 contactos de contactos, 442 probables contactos, incluidos 1985 trabajadores de la salud. Cuatro pacientes, uno confirmado y tres sospechosos de EVE actualmente se encuentran hospita- 
lizados en Centros de tratamiento de enfermedades epidémicas.

\section{República Democrática del Congo}

Al 4 de abril de 2021 no se han confirmado nuevos casos de EVE en el Norte de Kivu por 34 días consecutivos. Desde el comienzo del brote se han confirmado 11 casos, con un caso probable y seis muertes (letalidad del $50 \%$ ). Dos trabajadores de la salud se han contagiado y corresponden al $16,6 \%$ de los casos. De los 80 contactos listados al inicio del periodo de seguimiento de 42 días, 17 (21,2 \%) fueron encontrados, 39 (48,7\%) han completado su periodo de 42 días sin seguimiento y 24 todavía deben ser encontrados.

Al 4 de abril de 2021 se han reportado un total de 417 alertas, todas investigadas; de estas 37 fueron verificadas y 36 (97,3\%) fueron muestreadas. Actualmente no se encuentran casos confirmados de EVE en los centros de tratamiento para Ébola y 42 casos sospechosos reciben seguimiento en aislamiento en los establecimientos de salud.

Hasta el 4 de abril un total de 1898 personas han sido vacunadas, 1169 en Biena, 360 en Katwa, 297 en Butembo y 72 en Musienene. Desde el inicio de la epidemia 2952 muestras han sido recibidas y analizadas para EVE. Al 4 de abril 2021, un total de 12 alertas por muertes fueron reportadas, de estas, nueve recibieron entierros dignos y seguros, tres no fueron llevados a cabo porque los cuerpos aún permanecen en la morgue. Las actividades de prevención y control de infecciones continúan con apoyo en las 14 zonas de salud; 10000 litros de agua fueron entregados en el establecimiento de salud en Masoya y la población circundante.

Fuente: Organización Mundial de la Salud (OMS). Virus del Ébola. Boletín de brotes y emergencias semana epidemiológica 14. Fecha de publicación 4 de abril 2021. Fecha de consulta 8 de abril de 2021. Disponible en: https://www.afro.who.int/publications/outbreaks-and-emergenciesbulletin-week-14-29-march-04-april-2021

\section{SITUACIÓN COVID-19}

Hasta el 08 de abril de 2021 se han confirmado por laboratorio 2 492081 casos de COVID-19 en Colombia, en 38 entidades territoriales del orden departamental y distrital, afectando 1112 municipios. La incidencia acumulada es de 4 947,31 casos por cada 100 000 habitantes. A la fecha el 2,5\% (62 668) de los casos se encuentra activo, siendo procedente principalmente de Antioquia con el 32,0 \% (20 035), Bogotá con el 26,0 \% (16 298), Barranquilla D.E. con el 10,6\% (6 643), Valle del Cauca con el 7,3\% (4 551), Atlántico con el 4,4 \% (2 762) y Santander con el 2,4 (1 484). A nivel municipal, el 71,3\% (44691) de los casos activos se encuentra principalmente en Bogotá (16 298), Medellín (12 176), Barranquilla (6 643), Cali (3 361), Itagüí (1 861), Bello (1 739), Soledad (1 618) y Santa Marta (995).

La incidencia nacional de casos activos es de 124,41 casos por 100 000 habitantes. Los municipios con mayor incidencia de casos activos son: Itagüí - Antioquia (641,74), Rionegro - Antioquia $(547,46)$, Barranquilla DE (521,33), Caldas - Antioquia (484,12), Medellín Antioquia $(480,61)$, California - Santander $(358,42)$ y Envigado Antioquia $(346,83)$. De los casos activos el 51,9\% (32 507) se reportó en mujeres y según el grupo de edad el 70,4\% (44 146) se presenta en personas entre los 20 y los 59 años. De acuerdo con la ubicación, el 82,8 \% (51 883) de los casos activos se encuentra en casa, el 14,9\% (9 340) hospitalizado en sala general y el 2,3\% (1 445) hospitalizado en UCl.

Entre el 07 de marzo y el 03 de abril 2021 (SE 10 a 13, 2021), se reportaron 121529 casos de COVID-19, para una incidencia de 252,89 casos por 100000 habitantes. Las entidades territoriales que durante este periodo superan la incidencia de periodo nacional son Barranquilla (1 484,07), Santa Marta $(898,47)$, Atlántico $(654,49)$, Antioquia $(482,26)$, Bogotá $(325,26)$, Cali $(299,55)$ y Quindío $(273,22)$. Durante este periodo epidemiológico (SE 10 a 13, 2021) se presentó un aumento significativo de la incidencia 
respecto al periodo anterior en las entidades territoriales de Antioquia, Atlántico, Barranquilla, Caldas, Cesar, La Guajira, Santa Marta, Risaralda, Sucre, Archipiélago de San Andrés, Providencia y Santa Catalina y Vaupés. Por el contrario, las entidades territoriales de Bogotá $(0,9)$, Cali $(0,9)$, Quindío $(0,7)$ y Amazonas $(0,1)$, registraron un descenso significativo en la incidencia de COVID-19 en comparación con el periodo anterior (mapa 1).

Mapa 1. Incidencia de casos confirmados de COVID-19 por entidad territorial, Colombia, semana epidemiológica 10 a 13, 2021

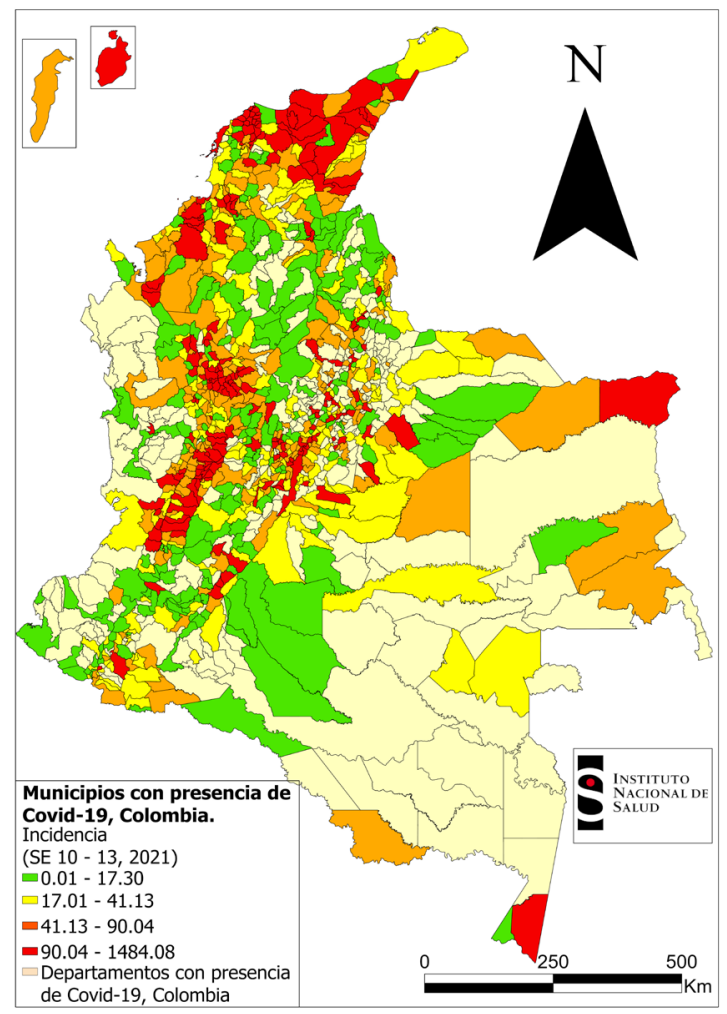

Fuente: INS- Sala de Análisis del Riesgo COVID19, 2021
Hasta la semana epidemiológica 13 se han reportado 63932 muertes, entre el 07 de marzo y el 03 de abril 2021 (SE 10 a 13, 2021), se notificaron 2980 fallecidos, lo que corresponde al 4,6\% del total de casos fatales. Las entidades con mayor notificación de fallecidos durante este periodo epidemiológico fueron: Antioquia con 15,7 \% (469), Bogotá con 14,1\% (420), Barranquilla con 10,4\% (310), Atlántico con 7,5 \% (224), Cali con 5,9 \% (176) y Córdoba con 4,9 \% (147). Entre la semana epidemiológica 10 a 13, 2021, los municipios con la mayor tasa de mortalidad fueron: Ciénaga - Magdalena $(70,93)$, Almeida - Boyacá $(55,65)$, Leticia - Amazonas $(52,34)$, Onzaga - Santander (49,31), Arbeláez - Cundinamarca $(45,80)$, Becerril - Cesar $(38,66)$ y Paya - Boyacá $(38,01)$ (mapa 2).

Mapa 2. Mortalidad por COVID-19 por entidad territorial, Colombia, semana epidemiológica 10 a 13, 2021

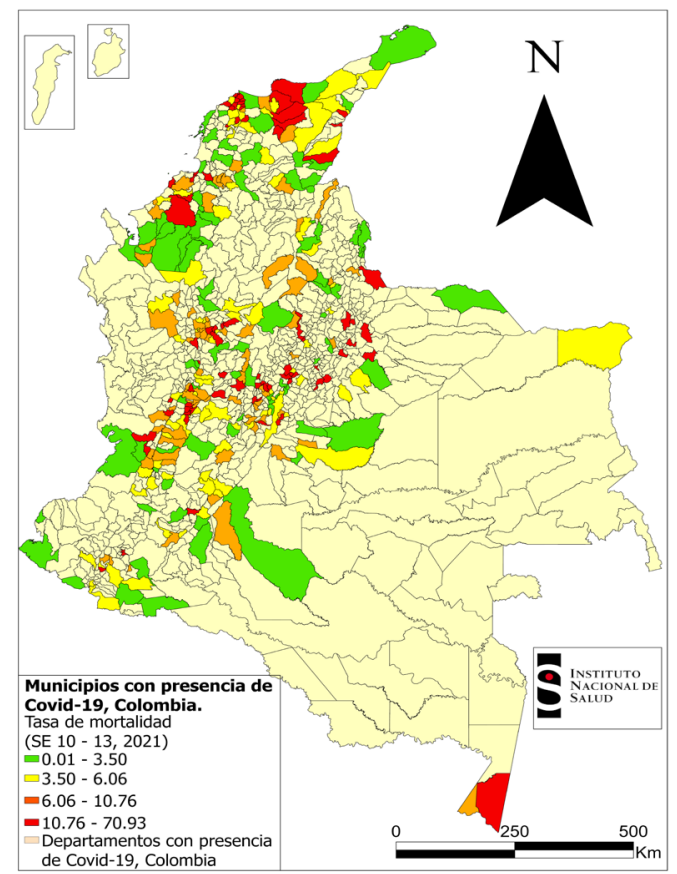

Fuente: INS- Sala de Análisis del Riesgo COVID19, 2021 
Durante el periodo analizado (SE 10 a 13, 2021) fallecieron 186 personas entre los 15 y 44 años y 2330 personas mayores de 60 años, que corresponden al 6,2 \% y 78,2 \% de las muertes del periodo. Al comparar la tasa de mortalidad y letalidad entre la SE 10 a 13 de 2021 con respecto al periodo anterior (SE 06 a 09), se observó que de los 316 municipios que reportaron muertes por COVID-19 durante el periodo analizado, el 29,1\% (92) presentó incremento significativo en la tasa de mortalidad y la letalidad.

Los municipios que presentaron mayor variación (aumento) en la tasa de mortalidad fueron Aracataca - Magdalena $(\Delta 5,0)$, Fundación - Magdalena $(\Delta 5,0)$, Fredonia - Antioquia $(\Delta 4,0)$, Moñitos Córdoba $(\Delta 4,0)$, Barbosa - Santander $(\Delta 4,0)$ y Cereté - Córdoba ( $\Delta$ $3,5)$. Adicionalmente, los municipios que presentaron mayor variación (aumento) en la tasa de letalidad fueron Onzaga - Santander $(\Delta 26,0)$, Moñitos - Córdoba $(\Delta 20,6)$, Convención - Norte de Santander $(\Delta 6,0)$, Fundación - Magdalena $(\Delta 4,8)$, Barbosa - Santander $(\Delta 4,6)$ y Coveñas - Sucre $(\Delta 4,4)$.

Durante el periodo analizado (SE 10 a 13, 2021), se han notificado por fecha de inicio de síntomas 49 conglomerados COVID-19, de los cuales el $100,0 \%$ se encuentra activo con 441 casos confirmados y 1765 muestras obtenidas para un porcentaje de positividad del 25,0\%. De los conglomerados activos, el 36,7\% (18) se presentó en empresas con 115 casos confirmados; 18,4\% (9) en centros de protección con 101 casos; $12,2 \%$ (6) en ámbito comunitario con 35 casos; 12,2 \% (6) en Fuerzas Militares con 92 casos; 6,1 \% (3) en instituciones educativas con 22 casos; 6,1\% (3) en instituciones de salud con 30 casos; $4,1 \%$ (2) en población carcelaria con 18 casos y $4,1 \%$ (2) en población indígena con 28 casos confirmados. 


\section{TABLAS DE MANDO NACIONAL}

Comportamiento de la notificación por departamento a semana 13
Decremento Incremento

\begin{tabular}{|c|c|c|c|c|c|c|c|c|c|c|c|c|c|c|c|c|c|c|}
\hline \multirow[b]{2}{*}{ Departamento } & \multicolumn{3}{|c|}{ Accidente ofídico } & \multicolumn{3}{|c|}{$\begin{array}{c}\text { Agresiones por animales } \\
\text { potencialmente transmisores de } \\
\text { rabia }\end{array}$} & \multicolumn{3}{|c|}{ Dengue } & \multicolumn{3}{|c|}{ EAPV } & \multicolumn{3}{|c|}{ Hepatitis A } & \multicolumn{3}{|c|}{ Intento de suicidio } \\
\hline & 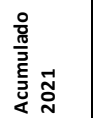 & 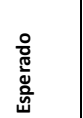 & 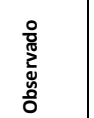 & 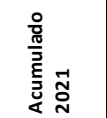 & 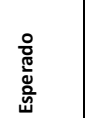 & 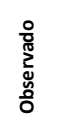 & 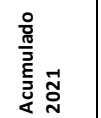 & 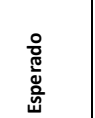 & 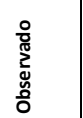 & 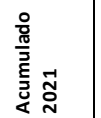 & 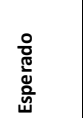 & 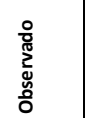 & 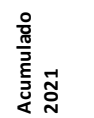 & 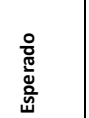 & 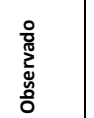 & 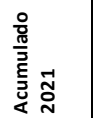 & 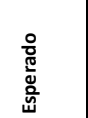 & 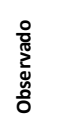 \\
\hline \begin{tabular}{|l|l|} 
Amazonas & \\
\end{tabular} & 7 & 1 & 0 & 30 & \begin{tabular}{l|l}
5 \\
\end{tabular} & 3 & 78 & 14 & 37 & 0 & 1 & 0 & 0 & 0 & 0 & 3 & 10 & 3 \\
\hline Antioquia & 131 & 11 & 4 & 3.216 & 255 & 219 & 366 & 290 & 128 & 35 & 2 & 0 & 36 & 25 & 4 & 981 & 1.362 & 981 \\
\hline Arauca & 25 & 2 & 1 & 157 & 13 & 15 & 50 & 37 & 20 & 0 & 0 & 0 & 12 & 1 & 2 & 31 & 62 & 31 \\
\hline Atlántico & 22 & 2 & 0 & 561 & 52 & 24 & 266 & 68 & 74 & 4 & 0 & 0 & 1 & 2 & 0 & 168 & 162 & 168 \\
\hline \begin{tabular}{|l} 
Barranquilla \\
\end{tabular} & 2 & 0 & 0 & 437 & 47 & 14 & 348 & 54 & 85 & 1 & 0 & 0 & $\frac{1}{4}$ & 2 & \begin{tabular}{l|l}
0 \\
\end{tabular} & 186 & 216 & 186 \\
\hline Bogotá & 0 & 0 & 0 & 4.070 & 367 & 276 & 0 & 0 & 0 & 76 & 2 & 0 & 5 & 10 & 2 & 899 & 870 & 899 \\
\hline Bolívar & 37 & 3 & 2 & 504 & 39 & 36 & 231 & 67 & 46 & \begin{tabular}{|l|}
3 \\
\end{tabular} & 0 & 0 & 0 & 1 & 0 & 105 & 118 & 105 \\
\hline \begin{tabular}{|l|} 
Boyacá \\
\end{tabular} & 26 & 1 & 1 & 985 & 96 & 88 & 104 & 48 & 34 & 9 & 1 & 1 & 1 & 2 & 0 & 114 & 193 & $\frac{114}{114}$ \\
\hline \begin{tabular}{|l} 
Buenaventura \\
\end{tabular} & 13 & 1 & 1 & 26 & 1 & 6 & 69 & 13 & 25 & 0 & 0 & 0 & 0 & 0 & 0 & 20 & 19 & 20 \\
\hline \begin{tabular}{|l} 
Caldas \\
\end{tabular} & 21 & 1 & 0 & 691 & 60 & 55 & 46 & 39 & 15 & 5 & 0 & 2 & 0 & 2 & 0 & 235 & 292 & 235 \\
\hline \begin{tabular}{|l|} 
Cali \\
\end{tabular} & 0 & 0 & 0 & 881 & 87 & 58 & 2.071 & 777 & 665 & 8 & 1 & 0 & 3 & 8 & \begin{tabular}{l|l}
1 \\
1
\end{tabular} & 314 & 408 & 314 \\
\hline Caquetá & 56 & 5 & 4 & 218 & 17 & 8 & 121 & 59 & 42 & 0 & 1 & 0 & 0 & 0 & 0 & 63 & 66 & 63 \\
\hline \begin{tabular}{|l|} 
Cartagena \\
\end{tabular} & 2 & 0 & 0 & 234 & 16 & 17 & 610 & 26 & 125 & 1 & 1 & 0 & 0 & 0 & \begin{tabular}{l|l}
0 \\
\end{tabular} & 108 & 142 & 108 \\
\hline \begin{tabular}{|l|} 
Casanare \\
\end{tabular} & 28 & 3 & 4 & 228 & 23 & 12 & 110 & 118 & 40 & 2 & 1 & 0 & 4 & 0 & 2 & 68 & 75 & 68 \\
\hline \begin{tabular}{|l} 
Cauca \\
\end{tabular} & 34 & 2 & 1 & 1.055 & 103 & 71 & 152 & 44 & 44 & 5 & 0 & 0 & 0 & 2 & \begin{tabular}{l|l} 
\\
0
\end{tabular} & 168 & 214 & $\begin{array}{r}168 \\
\end{array}$ \\
\hline Cesar & 40 & 3 & 3 & 415 & 34 & 17 & 291 & 135 & 115 & 1 & 0 & 0 & 0 & 2 & 0 & 152 & 176 & 152 \\
\hline Chocó & 43 & 4 & 1 & 34 & 1 & 1 & 99 & 16 & 31 & 0 & 0 & 0 & 0 & 0 & 0 & 10 & 32 & 10 \\
\hline \begin{tabular}{|l|} 
Córdoba \\
\end{tabular} & 31 & 4 & 1 & 817 & $\begin{array}{ll}63 \\
\end{array}$ & 41 & 166 & 125 & 57 & 5 & 3 & 0 & 0 & 2 & 0 & 185 & 220 & 185 \\
\hline \begin{tabular}{|l} 
Cundinamarca \\
\end{tabular} & 14 & 1 & 3 & 2.334 & 193 & 168 & 309 & 230 & 93 & 8 & 1 & 1 & 2 & 3 & \begin{tabular}{l|l}
1 \\
\end{tabular} & 366 & 465 & 366 \\
\hline \begin{tabular}{|l} 
Guainía \\
\end{tabular} & $\frac{14}{6}$ & $\frac{1}{0}$ & 0 & 26 & 2 & 1 & - & 3 & 0 & 0 & $\frac{1}{0}$ & $\begin{array}{ll}2 \\
\end{array}$ & $\frac{2}{0}$ & 0 & $\begin{array}{ll}1 \\
0\end{array}$ & 5 & 7 & 5 \\
\hline \begin{tabular}{|l|} 
Guaviare \\
\end{tabular} & 35 & 1 & 5 & 69 & 4 & 2 & 20 & 31 & 12 & 0 & 0 & 0 & 1 & 0 & \begin{tabular}{l|l}
0 \\
\end{tabular} & 8 & 17 & 8 \\
\hline \begin{tabular}{|l|} 
Huila \\
\end{tabular} & 25 & 2 & 1 & 1.013 & 66 & 93 & 419 & 333 & 172 & 6 & 0 & 1 & 0 & 1 & 0 & 182 & 191 & 182 \\
\hline La Guajira & 8 & 1 & 0 & 323 & 27 & 13 & 37 & 65 & 14 & 1 & 0 & 0 & 0 & 2 & 0 & 45 & 65 & 45 \\
\hline Magdalena & 25 & 2 & 1 & 448 & 46 & 33 & 188 & 30 & 51 & 3 & 1 & 1 & 0 & 1 & 0 & 75 & 66 & 75 \\
\hline Meta & 56 & 6 & 6 & 629 & 50 & 50 & 293 & 391 & 97 & 1 & 0 & 0 & 2 & 1 & 2 & 131 & 186 & 131 \\
\hline Nariño & 30 & 2 & 1 & 1.150 & 96 & 104 & 127 & 36 & 45 & 7 & 1 & 2 & 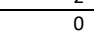 & 1 & \begin{tabular}{l|l}
0 \\
0
\end{tabular} & 250 & 324 & 250 \\
\hline Norte de Santander & 91 & 6 & 2 & 664 & 69 & 54 & 297 & 148 & 122 & 9 & 1 & 1 & 15 & 22 & \begin{tabular}{l|l}
3 \\
\end{tabular} & 203 & 214 & 203 \\
\hline \begin{tabular}{|l} 
Putumayo \\
\end{tabular} & 36 & 2 & $e_{0}^{2}$ & 257 & 21 & $\frac{34}{20}$ & 501 & 103 & 191 & 1 & $\frac{1}{0}$ & $\begin{array}{c}1 \\
0\end{array}$ & 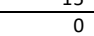 & $\frac{24}{1}$ & \begin{tabular}{l|l}
3 \\
0
\end{tabular} & 66 & 78 & $\frac{205}{66}$ \\
\hline \begin{tabular}{|l|} 
Quindío \\
\end{tabular} & 5 & 0 & 0 & 433 & 51 & 36 & 77 & 62 & 42 & 2 & 1 & 0 & 0 & 0 & 0 & 163 & 150 & 163 \\
\hline \begin{tabular}{|l|} 
Risaralda \\
\end{tabular} & 14 & 1 & 0 & 716 & 64 & 55 & 49 & 29 & 14 & 0 & 1 & 0 & 2 & 1 & 0 & 206 & 287 & 206 \\
\hline San Andrés & 0 & 0 & 0 & 26 & 5 & 4 & 26 & 3 & 8 & 0 & 0 & 0 & $\frac{2}{0}$ & 0 & 0 & 1 & 2 & $\frac{1}{1}$ \\
\hline Santa Marta D.E. & 1 & 1 & 0 & 256 & 35 & 16 & 136 & 21 & 23 & 3 & 1 & 0 & 0 & 0 & \begin{tabular}{l|l}
0 \\
\end{tabular} & 53 & 75 & 53 \\
\hline Santander & 39 & 4 & 1 & 1.051 & 91 & 77 & 218 & 468 & 83 & 4 & 1 & 0 & 2 & 4 & \begin{tabular}{l|l}
0 \\
\end{tabular} & 291 & 273 & 291 \\
\hline Sucre & 14 & 2 & 0 & 487 & 45 & 22 & 175 & 117 & 58 & 5 & 2 & 1 & 0 & 1 & \begin{tabular}{l|l}
0 \\
0
\end{tabular} & 81 & 112 & 81 \\
\hline Tolima & 31 & 3 & 1 & 976 & 85 & 94 & 415 & 572 & 134 & \begin{tabular}{l|l|}
16 \\
\end{tabular} & 0 & 0 & 2 & 2 & \begin{tabular}{l|l}
1 \\
\end{tabular} & 256 & 345 & 256 \\
\hline Valle del Cauca & 12 & 1 & 0 & 1.311 & 119 & 105 & 734 & 359 & 250 & 8 & 0 & 0 & 1 & 2 & 0 & 273 & 374 & 273 \\
\hline \begin{tabular}{|l|} 
Vaupés \\
\end{tabular} & 13 & 0 & 1 & 42 & 3 & 0 & 0 & 1 & 0 & 0 & 0 & 0 & 0 & 0 & 0 & 3 & 6 & 3 \\
\hline Vichada & 10 & 1 & 3 & 57 & 3 & 2 & 10 & 10 & 1 & 0 & 0 & 0 & 0 & 0 & 0 & 1 & 5 & 1 \\
\hline Total nacional & 983 & 78 & 48 & 26.827 & 2.353 & 1.910 & 9.215 & 4.942 & 2.993 & 229 & 23 & 10 & 93 & 98 & 18 & 6.469 & 7.879 & 6.469 \\
\hline
\end{tabular}

Tema central

Situación Nal.

Mortalidad

Trazadores

Brotes

COVID-19

Tablas 


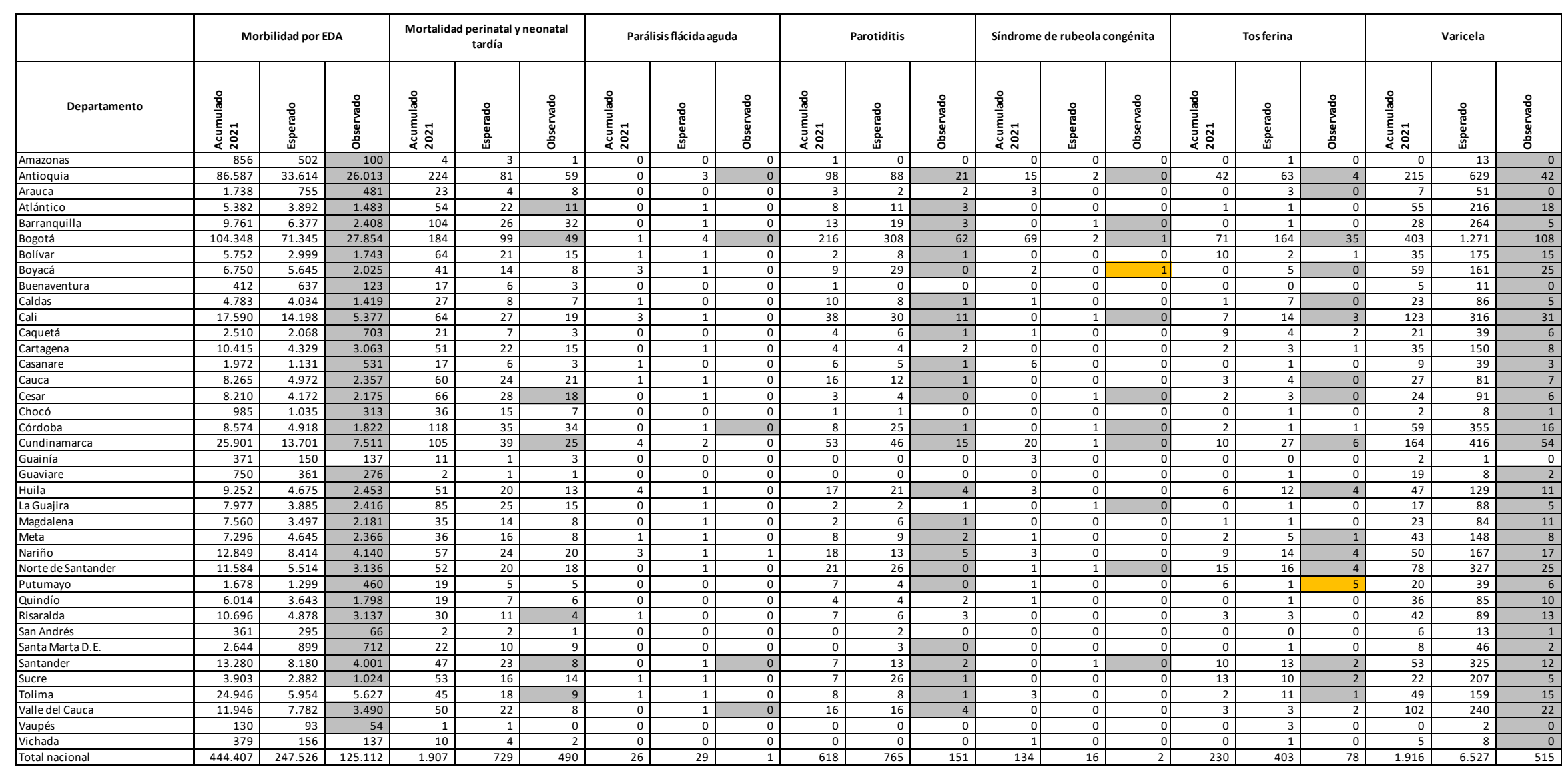

Tema central

Situación Nal.

Mortalidad

Trazadores

Brotes

COVID-19

Tablas 


\section{Comportamiento de la notificación por departamento a semana 13}

Decremento

Incremento

\begin{tabular}{|c|c|c|c|c|c|c|c|c|c|c|c|c|c|c|c|c|}
\hline \multirow[b]{2}{*}{ Departamento } & \multicolumn{2}{|c|}{ Chagas agudo } & \multicolumn{2}{|c|}{ Chikungunya } & \multicolumn{2}{|c|}{ Difteria } & \multicolumn{2}{|c|}{$\begin{array}{c}\text { Enfermedad por virus } \\
\text { Zika }\end{array}$} & \multicolumn{2}{|c|}{ Leishmaniasis } & \multicolumn{2}{|c|}{ Leptospirosis } & \multicolumn{2}{|c|}{ Mortalidad materna } & \multicolumn{2}{|c|}{$\begin{array}{c}\text { Mortalidad por IRA en } \\
\text { menores de } 5 \text { años }\end{array}$} \\
\hline & 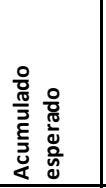 & 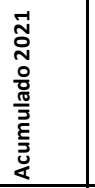 & $\begin{array}{l}\frac{0}{0} \frac{\pi}{5} \\
\frac{\pi}{5} \\
\frac{\pi}{0} \\
\bar{y} \\
\frac{0}{4}\end{array}$ & 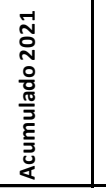 & 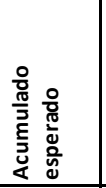 & 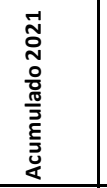 & 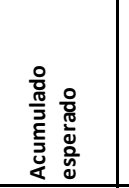 & 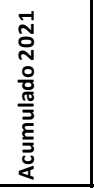 & 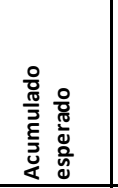 & 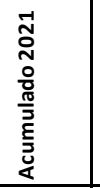 & 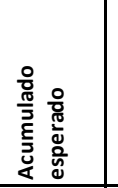 & 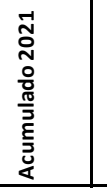 & 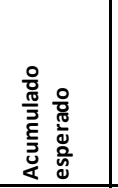 & 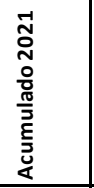 & 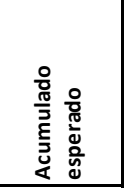 & 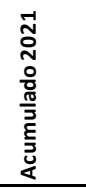 \\
\hline \begin{tabular}{|l|l|} 
Amazonas \\
\end{tabular} & 0 & 0 & 3 & 0 & 0 & 0 & 0 & 0 & 4 & 3 & 0 & \begin{tabular}{l|l}
1 \\
\end{tabular} & 1 & 0 & 1 & 1 \\
\hline \begin{tabular}{|l|} 
Antioquia \\
\end{tabular} & 0 & 0 & 11 | & 4 & 0 & 0 & 3 & 5 & 465 & 160 & 75 & 88 & 7 & 11 & 18 & 12 \\
\hline Arauca & 0 & 0 & $2 \mid$ & \begin{tabular}{l|l}
0 \\
\end{tabular} & 0 & 0 & $2 \mid$ & 1 & 5 & 5 & 0 & 1 & 0 & 0 & 0 & 1 \\
\hline Atlántico & 0 & 0 & $2 \mid$ & \begin{tabular}{l|l}
0 \\
\end{tabular} & 0 & 0 & $2 \mid$ & 2 & 1 & 0 & 13 & \begin{tabular}{l|l|}
11 \\
\end{tabular} & 3 & 1 & 1 & 2 \\
\hline \begin{tabular}{|l|} 
Barranquilla \\
\end{tabular} & 0 & 0 & 6 & \begin{tabular}{l|l}
0 \\
\end{tabular} & 0 & 0 & 2 2 & 3 & 1 & 0 & 12 & 23 & 2 & 3 & 2 & 1 \\
\hline Bogotá & 1 & 0 & 0 & 0 & 0 & 0 & 1 & 7 & 0 & 0 & 15 & 21 & 5 & 9 & 8 & 8 \\
\hline Bolívar & 0 & 0 & $2 \mid$ & \begin{tabular}{l|l}
0 \\
\end{tabular} & 0 & 0 & $1 \mid$ & 0 & 114 & 40 & 11 & \begin{tabular}{l|l}
5 \\
\end{tabular} & 2 & 3 & 4 & 4 \\
\hline Boyacá & 1 & 0 & 1 & 1 & 0 & 0 & 1 & 0 & 42 & 11 & 2 & \begin{tabular}{l|l}
5 \\
\end{tabular} & 2 & 0 & 1 & 1 \\
\hline Buenaventura & 0 & 0 & $1 \mid$ & \begin{tabular}{l|l}
0 \\
\end{tabular} & 0 & 0 & 0 & 0 & 8 & 2 & 10 & 21 & 1 & 1 & 1 & 0 \\
\hline Caldas & 0 & 0 & $2 \mid$ & \begin{tabular}{l|l}
0 \\
\end{tabular} & 0 & 0 & 0 & 0 & 54 & 60 & 4 & \begin{tabular}{l|l}
4 \\
\end{tabular} & 1 & 1 & 1 & 1 \\
\hline Cali & 0 & 0 & 32 & \begin{tabular}{l|l}
1 \\
\end{tabular} & 0 & 0 & 22 & 3 & 1 & 1 & 21 & \begin{tabular}{l|l}
46 & \\
\end{tabular} & 2 & 5 & 3 & 0 \\
\hline Caquetá & 0 & 1 & $3 \mid$ & 0 & 0 & 0 & \begin{tabular}{l|l}
3 & \\
\end{tabular} & 0 & 86 & 27 & \begin{tabular}{l|l|}
5 & \\
\end{tabular} & 2 & 1 & 0 & 1 & 2 \\
\hline \begin{tabular}{|l|} 
Cartagena \\
\end{tabular} & 0 & 0 & 4 & 0 & 0 & 0 & 0 & 0 & 0 & 1 & 3 & \begin{tabular}{l|l}
9 \\
\end{tabular} & 2 & 2 & 3 & 1 \\
\hline Casanare & 6 & 1 & $7 \mid$ & 0 & 0 & 0 & 1 & 0 & 4 & 1 & 1 & 2 & 1 & 0 & 1 & 0 \\
\hline Cauca & 0 & 0 & $1 \mid$ & 0 & 0 & 0 & \begin{tabular}{l|l}
1 & \\
\end{tabular} & 0 & 27 & 7 & \begin{tabular}{l|l}
9 \\
\end{tabular} & 7 & 3 & 0 & 2 & 2 \\
\hline Cesar & 2 & 0 & 3 & 0 & 0 & 0 & 2 & 0 & 14 & 5 & 7 & \begin{tabular}{l|l}
6 \\
\end{tabular} & 3 & 2 & 3 & 3 \\
\hline Chocó & 0 & 0 & 0 & 0 & 0 & 0 & 0 & 0 & 84 & 58 & \begin{tabular}{l|l}
9 \\
\end{tabular} & \begin{tabular}{l|l}
6 & \\
\end{tabular} & 4 & 3 & 9 & 8 \\
\hline Córdoba & 0 & 0 & $2 \mid$ & \begin{tabular}{l|l}
0 \\
\end{tabular} & 0 & 0 & $2 \mid$ & 1 & 44 & 16 & \begin{tabular}{l|l}
3 \\
\end{tabular} & \begin{tabular}{l|l}
12 \\
\end{tabular} & 3 & 5 & 3 & 2 \\
\hline Cundinamarca & 1 & 0 & 21 & 2 & 0 & 0 & \begin{tabular}{l|l}
9 \\
\end{tabular} & 4 & 68 & 13 & 15 & \begin{tabular}{l|l}
11 \\
\end{tabular} & 4 & 5 & 5 & 2 \\
\hline Guainía & 0 & 0 & 0 & \begin{tabular}{l|l}
0 \\
\end{tabular} & 0 & 0 & 0 & 0 & 16 & 1 & \begin{tabular}{l|l}
7 \\
\end{tabular} & \begin{tabular}{l|l}
0 \\
\end{tabular} & 0 & 0 & 0 & 2 \\
\hline Guaviare & 0 & 0 & 9 & \begin{tabular}{l|l}
0 \\
\end{tabular} & 0 & 0 & \begin{tabular}{l|l}
1 & \\
\end{tabular} & 0 & 112 & 70 & \begin{tabular}{l|l}
2 \\
\end{tabular} & 2 & 0 & 1 & 0 & 0 \\
\hline \begin{tabular}{|l|} 
Huila \\
\end{tabular} & 0 & 0 & \begin{tabular}{l|l}
15 \\
\end{tabular} & \begin{tabular}{l|l}
0 \\
\end{tabular} & 0 & 0 & $2 \mid$ & 1 & 11 & 1 & 28 & \begin{tabular}{l|l|}
15 & \\
1
\end{tabular} & 0 & 2 & 1 & 2 \\
\hline \begin{tabular}{|l|} 
La Guajira \\
\end{tabular} & 0 & 0 & 0 & 0 & 0 & 0 & 定 & 0 & 19 & 8 & 0 & 1 & 7 & 4 & 9 & 7 \\
\hline Magdalena & 0 & 0 & $1 \mid$ & \begin{tabular}{l|l}
0 \\
\end{tabular} & 0 & 0 & 0 & 1 & 2 & 1 & 1 & 2 & 4 & 7 & 4 & 2 \\
\hline Meta & 1 & 0 & 13 & \begin{tabular}{l|l}
0 \\
\end{tabular} & 0 & 0 & $2 \mid$ & 4 & 114 & 37 & $3 \mid$ & 8 & 3 & 5 & 2 & 2 \\
\hline Nariño & 0 & 0 & 0 & 0 & 0 & 0 & 1 & 2 & 165 & 62 & 1 1 & 8 & 5 & 1 & 4 & 1 \\
\hline Norte de Santander & 0 & 0 & 6 & 0 & 1 & 0 & 32 & 1 & 151 & 43 & $7 \mid$ & 2 & 3 & 3 & 3 & 3 \\
\hline \begin{tabular}{|l|} 
Putumayo \\
\end{tabular} & 0 & 0 & 18 & 1 & 0 & 0 & 34 & 0 & 49 & 26 & $2 \mid$ & \begin{tabular}{l|l}
4 \\
\end{tabular} & 1 & 1 & 2 & 0 \\
\hline Quindío & 0 & 0 & \begin{tabular}{l|l}
3 & \\
\end{tabular} & \begin{tabular}{l|l}
0 \\
\end{tabular} & 0 & 0 & 0 & 0 & 0 & 1 & 16 & 21 & 0 & 0 & 1 & 0 \\
\hline Risaralda & 0 & 0 & \begin{tabular}{l|l}
9 \\
\end{tabular} & 0 & 0 & 0 & $3 \mid$ & 1 & 70 & 10 & 54 & \begin{tabular}{l|l}
35 \\
\end{tabular} & 2 & 3 & 1 & 2 \\
\hline San Andrés & 0 & 0 & 0 & 0 & 0 & 0 & 0 & 0 & 0 & 0 & \begin{tabular}{l|l}
0 \\
\end{tabular} & \begin{tabular}{l|l}
0 & 1 \\
\end{tabular} & 0 & 0 & 0 & 0 \\
\hline Santa Marta D.E. & 0 & 0 & $2 \mid$ & \begin{tabular}{l|l}
0 \\
\end{tabular} & 0 & 0 & \begin{tabular}{l|l}
1 & \\
\end{tabular} & 0 & 4 & 1 & 0 & \begin{tabular}{l|l}
1 & \\
\end{tabular} & 2 & 4 & 1 & 1 \\
\hline Santander & 2 & 0 & 23 & 1 & 0 & 0 & \begin{tabular}{l|l}
9 \\
\end{tabular} & 2 & 221 & 88 & 11 & \begin{tabular}{l|l}
15 \\
\end{tabular} & 2 & 2 & 2 & 4 \\
\hline Sucre & 0 & 0 & $1 \mid$ & 0 & 0 & 0 & $1 \mid$ & 0 & 16 & 21 & 7 & \begin{tabular}{l|l}
9 & 0 \\
9 &
\end{tabular} & 2 & 2 & 3 & 1 \\
\hline Tolima & 1 & 0 & $17 \mid$ & 1 & 0 & 0 & 9 & 1 & 306 & 32 & 62 & 50 & 2 & 2 & 4 & 4 \\
\hline Valle del Cauca & 0 & 0 & 5 & 2 & 0 & 0 & 20 & 0 & 12 & 7 & \begin{tabular}{l|l|}
97 \\
\end{tabular} & 30 & 1 & 4 & 2 & 4 \\
\hline Vaupés & 0 & 0 & 0 & 0 & 0 & 0 & 0 & 0 & 12 & 5 & 0 & 0 & 0 & 0 & 0 & 0 \\
\hline Vichada & 0 & 0 & 0 & \begin{tabular}{l|l}
0 \\
\end{tabular} & 0 & 0 & 1 & 0 & 14 & 7 & \begin{tabular}{l|l}
1 \\
\end{tabular} & 0 & 1 & 0 & 1 & 0 \\
\hline \begin{tabular}{|l|} 
Total nacional \\
\end{tabular} & \begin{tabular}{|l|l}
15 & \\
\end{tabular} & 2 & 225 & 13 & \begin{tabular}{l|l}
1 & \\
\end{tabular} & 0 & \begin{tabular}{|l|l|}
167 \\
\end{tabular} & \begin{tabular}{|l|l|}
39 \\
\end{tabular} & \begin{tabular}{l|l}
2.316 \\
\end{tabular} & 831 & \begin{tabular}{|l|l|}
514 & \\
\end{tabular} & \begin{tabular}{l|l|}
484 \\
\end{tabular} & \begin{tabular}{l|l}
82 & \\
\end{tabular} & \begin{tabular}{l|l|}
92 & \\
\end{tabular} & 107 & 86 \\
\hline
\end{tabular}




\section{Semana epidemiológica 13}

28 de marzo al 3 de abril de 2021

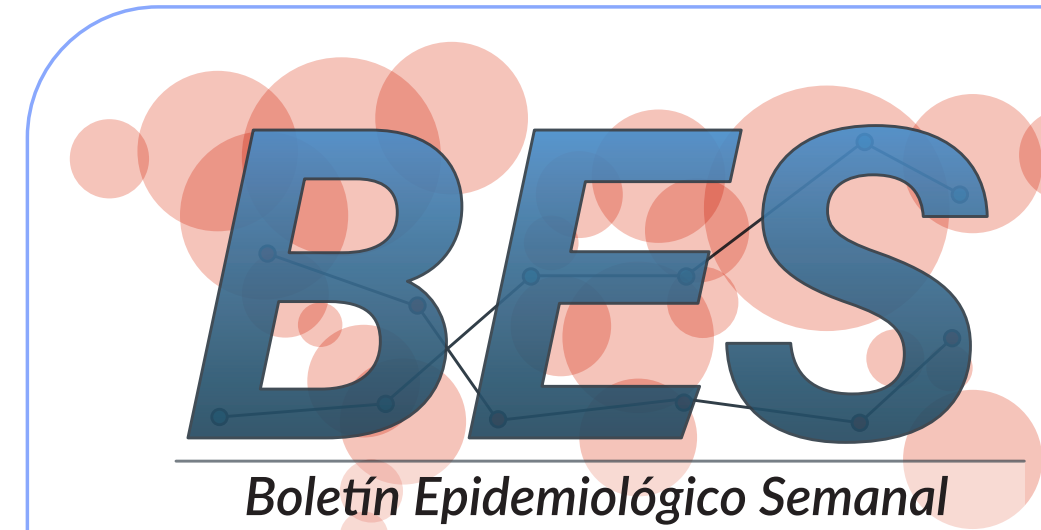

\section{Boletín Epidemiológico Semanal}

\section{Expertos Temáticos}

Situación Nacional

Grupo Sivigila sivigila@ins.gov.co

Mortalidad

Giana María Henriquez Mendoza ghenriquez@ins.gov.co

María Eugenia Pinilla Saraza mpinillas@ins.gov.co

Dra. Diana Walteros

Dr. Hernán Quijada Bonilla

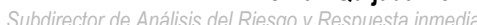

Una publicación del

Instituto Nacional de Salud Dirección de Vigilancia y Análisis del Riesgo en Salud Pública Publicación en línea: ISSN 2357-6189 https://doi.org/10.33610/23576189.2021.13

Asistencia técnica de:

Bloomberg Philanthropies $>$ HEALTH INITIATIVE
Eventos Trazadores

\section{Andrea Jineth Rodriguez Reyes arodriguezr@ins.gov.co Sandra Milena Aparicio Fuentes saparicio@ins.gov.co}

Carolina Ferro Mendez cferro@ins.gov.co

Brotes

Gestor sistema de alerta temprana eri@ins gov.co

Angela Patricia Alarcón aalarcon@ins.gov.co

Tablas de mando

Yudy Silva Lizarazoysilva@ins.gov.co

Diana Rivera drivera@ins.gov.co
Dr. Hernán Quijada Bonilla hquijada@ins.gov.co

Diseño y diagramación

Alexander Casas acasasc@ins gov.co
La salud

es de todos 